\title{
M82, Starbursts, Star Clusters, and the formation of Globular Clusters
}

\author{
Eric Keto ${ }^{1}$, Luis C. Ho ${ }^{2}$, and K.-Y. Lo ${ }^{3}$
}

\begin{abstract}
We observed the nearby starburst galaxy M82 in CO in the higher frequency $(2-1)$ transition to achieve an angular resolution below 1 arc second or $17 \mathrm{pc}$ at the target. We resolved the molecular gas into a large number of compact clouds, with masses ranging from $\sim 2 \times 10^{3}$ to $2 \times 10^{6} M_{\odot}$. The mass spectrum scales as $N(M) \propto M^{-1.5 \pm 0.1}$, similar to the mass spectra of young massive star clusters suggesting that individual molecular clouds are transformed in the starburst into individual star clusters. The larger clouds are surrounded by supernovae and HII regions suggesting that star formation proceeds from the outside of the clouds and progresses inward consistent with triggering by a sudden increase in external pressure. The clouds with internal star formation have velocity gradients and inverse P-Cygni spectral line profiles indicating inward motions of $35 \mathrm{kms}^{-1}$ consistent with shock driven compression. Diffuse free-free radio emission and X-ray emission around the clouds provides evidence for superheated ionized gas sufficient to drive the compression. Clouds with spectral lines indicating expansion show little internal star formation suggesting that the dynamics precedes and is responsible for the star formation rather than the inverse. M82 is known to be in interaction with neighboring M81. The overall picture is consistent with the formation of massive star clusters from individual giant molecular clouds crushed by a sudden galactic scale increase in external pressure generated by the changing dynamics that result from a near-collision with a neighboring galaxy. Present day globular clusters may have formed in a similar fashion in primordial galaxies.
\end{abstract}

Subject headings: galaxies: individual (M82) — galaxies: ISM — galaxies: starburst - galaxies: star clusters — ISM: clouds

\footnotetext{
${ }^{1}$ Harvard-Smithsonian Center for Astrophysics, 60 Garden Street, Cambridge, MA 02138

${ }^{2}$ The Observatories of the Carnegie Institution of Washington, 813 Santa Barbara Street, Pasadena, CA 91101

${ }^{3}$ National Radio Astronomy Observatory, 520 Edgemont Road, Charlottesville, VA 22903-2475
} 


\section{Introduction}

Two of the many interesting phenomena discovered by the satellite observatories have been the starburst galaxies and the super star clusters, first identified as important classes by the IRAS and the Hubble Space Telescope (HST) satellites (Soifer et al 1987; Holtzmann et al. 1992). The starburst galaxies are characterized by extremely rapid star formation in regions with gas densities up to 20 times higher than the typical densities of normal spiral galaxies (Sanders \& Mirabel 1996). Most starbursts are found in interacting galaxies, and the dynamics of the interaction may be responsible for the high concentration of gas and dust (Sanders \& Mirabel 1996; Kumai, Hashi \& Fujimoto 1993) that both provides the raw material for star formation and also obscures and downconverts most of the light of the starburst into the infrared.

In areas of lower extinction between the dust lanes of the starbursts, recent HST observations have revealed swarms of bright compact star clusters termed "super star clusters" or SSCs. The luminosity of these clusters comprises a significant fraction of the optical and near infrared light emitted in starbursts (Barth et al. 1995; Meurer et al. 1995; Maoz et al. 1996, 2001; Ho 1997) leading to the inference that the SSCs may be the dominant mode of star formation in starburst regions (Ho 1997). The HST observations show that SSCs represent perhaps the most extreme mode of star formation known. The SSCs are characterized by their high luminosities, from 1-100 times that of the R136 cluster of 30 Doradus in the Large Magellanic Clouds, exceptional compactness (half-light radii $\leq 2-5 \mathrm{pc}$ ) and young ages ( $\lesssim$ few hundred Myr old). Observations of the internal kinematics of some SSCs ((Ho \& Filippenko 1996a,b; Mengel et al. 2002; Larsen \& Richtler 2004) have shown that these examples are extremely massive $\left(z 10^{5} M_{\odot}\right)$ and most likely gravitationally bound. Estimates of SSC masses based on population synthesis models indicate a range from below $10^{4}$ up to a few $10^{6} \mathrm{M}_{\odot}$ (Zhang \& Fall 1999; Melo et al. 2005). The similarity in size and

mass of the SSCs and globular clusters have prompted the further suggestion that the SSCs might be present-day analogs of young globular clusters (Holtzmann et al. 1992). Previously thought to form strictly in an earlier epoch of galaxy evolution, globular clusters may in fact be forming in the current epoch in starburst environments (Ashman \& Zepf 2001).

The observations suggest a fascinating connection between starbursts, SSCs, and globular clusters, yet many questions remain. For example, what sets the mass scale of the globular clusters? The mass spectrum of globular clusters is peaked around $10^{5} \mathrm{M}_{\odot}$. Previously this was thought to be an observation of particular significance indicating a preferred mass scale at the primordial epoch of globular cluster formation (Peebles \& Dicke 1968; Fall \& Rees 1985). Did the globular clusters form in the past in the same way as we expect they may be forming today, from SSCs in starbursts? Is there a dominant mass scale in starbursts? 
Observations of the luminosity of SSCs, translatable to mass by means of population synthesis models, nominally show a peaked distribution similar to the globular cluster distribution (Zhang \& Fall 1999; Melo et al. 2005). However, the lowest luminosity clusters are not fully detectable by the HST (Zhang \& Fall 1999; Whitmore et al. 1999) and best efforts to correct the incompleteness at the low end of the mass spectrum result in a power law spectrum rather than a peaked spectrum. These observations suggest the hypothesis that the ancient globular clusters formed with a scale-free power law mass spectrum, the same as the SSCs, and the globular clusters acquired their present characteristic mass after billions of years of evolution has destroyed the low mass end of the original power law (Aguilar, Hut, Ostriker 1988; Chernoff \& Weinberg 1990; Vesperini et al. 2003).

The mass spectrum of globular clusters at the upper end where completeness is not a question has a slope of -1.7 (Harris \& Pudritz 1994), characteristic of molecular clouds in our Galaxy. The similarity of the cloud and cluster mass spectra suggests that it may be the cloud mass spectrum that determines the masses of the clusters. For example, SSCs might form from the rapid transformation of entire individual molecular clouds into stars facilitated by the extreme environment of a starburst. This would be as expected from simple theoretical considerations that suggest that if a gravitationally bound molecular cloud with an equilibrium internal velocity dispersion evolves to a stellar cluster with a similar velocity dispersion, then most of the cloud mass must be converted to stars (Elmegreen 1983; Lada, Margulis, \& Dearborn 1984) if the cluster is to remain gravitationally bound after the loss of whatever gas was not transformed into stars.

In addition to the possible link between starbursts, SSCs and globular clusters, many questions hinge on the determination of the mass spectrum of the SSCs and the molecular clouds in starbursts. Do SSCs form from the collapse of individual molecular clouds (Schweizer et al. 1996; Ashman \& Zepf 2001) or do the SSCs form as cores in the high densities within super-giant molecular clouds (Harris \& Pudritz 1994; Wilson et al. 2003). Is this question properly posed? For example, if the mass spectrum of clouds is scale free, there may be no distinction between super-giant molecular clouds and a closely packed collection of more normal giant molecular clouds. Is star formation in starbursts triggered by galactic scale shocks that result from the galaxy interactions associated with most starbursts. Is star formation sequential? Is the high star formation rate in starbursts simply scaled up by the high gas density as indicated by the universality of the Schmidt law (Schmidt 1959; Kennicutt 1998)? Is there a universal mode of star formation in open and bound clusters in our Galaxy and in SSCs in starbursts such that star formation in SSCs is a scaled up version of star formation in our own Galaxy (Elmegreen \& Efremov 1997). If so does this suggest a universal stellar initial mass function for all star formation environments. 
The number of fundamental questions affected by the mass spectrum of molecular clouds in starbursts motivates an observational determination. Molecular line observations indicate that the molecular clouds in starbursts are also distributed with a -1.5 power law spectrum. However, the identification of individual clouds at the lower end of the cloud mass spectrum is limited by the angular resolution of the current generation of millimeter wave interferometers. Therefore the determination of the cloud mass spectrum, similar to the cluster mass spectrum, is also troubled by the completeness of the sample. New molecular line observations at higher angular resolution are required to obtain a more complete sample at the lower end of cloud mass spectrum.

To improve on the existing molecular line observations requires higher angular resolution. This is achievable with the same millimeter interferometers used in previous observations if the new observations are made at the highest operating frequencies. For example, the $\mathrm{CO}(2-1)$ transition at $231 \mathrm{GHz}$ obtains an angular resolution (just under one arc second at the Owens Valley Radio Observatory) that is twice as good as that achieved by the same interferometer when observing the ground state transitions of CO and other molecules around $100 \mathrm{GHz}$.

We identified the starburst galaxy M82 as a suitable candidate for an observational determination of the mass spectrum of the interstellar medium in a starburst. First, this galaxy is sufficiently nearby that with arc second angular resolution we can resolve the ISM to a linear scale comparable to the SSC dimensions. M82 lies at the same distance as M81, 3.6 Mpc, which has been accurately determined based on observations of Cepheids (Freedman et al. 1994). At this distance, $1^{\prime \prime}$ corresponds to a linear size of $17 \mathrm{pc}$, only a factor of 2 larger than the typical diameters $(6-10 \mathrm{pc})$ of the SSCs. It is important to reach the cluster scale for two reasons. First the range of the mass spectrum of clouds that we observe should be comparable to that observed in clusters particularly at the critical lower end. (The relationship between the mass and length scales is understood by considering that if an individual molecular cloud is transformed by fragmentation to stars at the high efficiencies required to produce a bound cluster, the length scales of the precursor molecular cloud and the resulting cluster will be comparable.) Secondly, observations at a linear scale of $50-100 \mathrm{pc}$ are unable to determine whether a molecular cloud of cluster mass has a density that is the same as a typical molecular cloud in our own Galaxy, or if the same mass is concentrated in a smaller cloud of cluster scale dimensions with a density 100 to 1000 times higher, as would be expected from the high average surface density in the starburst environment.

Second, M82 has a history of extensive SSC production, with major episodes of past cluster formation and evidence for continued, ongoing cluster formation. HST observa- 
tions have identified an earlier epoch of cluster formation in the region known as M82B. (de Grijs, O’Connell, \& Gallagher 2001; McCrady, Gilbert, \& Graham 2003; Melo et al. 2005; O'Connell et al. 1995). Current cluster formation appears to be taking place in a nearby gas rich region where lower angular resolution observations $\left(>2.5^{\prime \prime} \approx 43 \mathrm{pc}\right)$ indicate a large number of discrete clouds with a power-law mass spectrum (Brouillet \& Schilke 1993; Shen \& Lo 1995). M82 is sufficiently gas-rich that it has the potential that the present-day ISM might reflect, at least statistically, the initial conditions of the precursor clouds that might collapse to form SSCs.

While the observations were primarily motivated by the question of the mass spectrum of the molecular clouds, a comparison of the distribution and dynamics of the molecular clouds with the locations of star formation activity as indicated by other observations, suggests several interesting hypotheses about star bursts and the formation of massive star clusters. The observations suggest that star formation begins in the outer regions of the clouds rather than their centers. This is different than star formation in our own Galaxy where we find star formation associated primarily with the centers of molecular clouds where, because of the self-gravity of the clouds, the gas density is highest. A comparison of star formation activity and the gas velocities in the clouds in M82, as revealed by the spectral information in our observations, shows that star formation occurs where the velocity fields indicate massive compression on the scale of the giant molecular clouds. Theoretical considerations then lead to a hypothesis for star formation in star bursts triggered by large scale shocks driven into the molecular clouds by a sudden increase in the external pressure. This mode of star formation is fundamentally different from normal star formation in our own Galaxy that takes place more slowly presumably motivated by a gradual increase in the density and pressure of the ISM or the slow loss of the internal energy of molecular clouds.

\section{Observations and Data Analysis}

The observations were made using the Millimeter Array at the Owens Valley Radio Observatory (OVRO). Approximately 64 hours of data were collected on a number of dates during the winter seasons of 1999 and 2000. Data from two phase centers, the left and right sides of the galaxy located at $(\alpha, \delta)_{\mathrm{J} 2000}=\left(09^{h} 55^{m} 53.19^{s}, 69^{\circ} 40^{\prime} 51.9^{\prime \prime}\right)$ and $\left(09^{h} 55^{m} 48.33^{s}\right.$, $\left.69^{\circ} 40^{\prime} 44.0^{\prime \prime}\right)$, respectively, were combined to form an image of the central $1 \times 0.5 \mathrm{kpc}$ of M82 made up of 2 overlapping beams of $25^{\prime \prime}$ diameter separated by $25^{\prime \prime}$. The correlator was tuned to $230.53799 \mathrm{GHz}$, the rest frequency of $\mathrm{CO}(2-1)$, with a spectral resolution of $4 \mathrm{MHz}(\sim 5$ $\mathrm{km} \mathrm{s}^{-1}$ ) in 128 frequencies. The channels were Hanning smoothed in the data processing to achieve a final spectral resolution of $10.4 \mathrm{kms}^{-1}$ per channel. Baselines ranged from 20 to 
$180 \mathrm{k} \lambda$ covering the range of spatial scales from approximately $1^{\prime \prime}$ to $10^{\prime \prime}$ or 17 to $170 \mathrm{pc}$ in linear scale at the distance of M82. Calibration, done in the MIR data reduction package developed at OVRO and the Submillimeter Array, used the Seyfert galaxies NGC 1275 (3C 84) and 0923+392 (4C 39.25) for amplitude and gain calibration, and the quasar 3C 273 for bandpass calibration. Mapping was done with the NRAO AIPS software. The FWHM of the synthesized beam was $1.0^{\prime \prime} \times 0.8^{\prime \prime}$, and the rms noise per channel was $0.01 \mathrm{Jy} \mathrm{beam}^{-1}$.

The integrated intensity of the $\mathrm{CO}(2-1)$ emission above a threshold of twice the rms noise or $2 \sigma=0.02 \mathrm{Jy}_{\text {beam }^{-1}}$ is shown in figure 1 . The map shows a number of large cloud complexes along the major axis of the galaxy, which is viewed nearly edge-on. The smallest clumps depicted in the map are unresolved.

The structures seen in our high resolution observations of $\mathrm{CO}(2-1)$ are consistent with the structures seen in previous molecular line observations, but there are differences. Because our observations were made at high angular resolution with an interferometer sensitive to a limited range of spatial scales, they do not have the same sensitivity to large scale diffuse structure as previous observations made at lower angular resolution. Secondly, the spatial distribution of $\mathrm{CO}(2-1)$ more closely matches $\mathrm{HCN}(1-0)$ (Brouillet \& Schilke 1993), than CO(1-0) (Shen \& Lo 1995; Matsushita et al. 2005). Because HCN has a higher critical density for excitation than $\mathrm{CO}(1-0)$, the comparison suggests that the $\mathrm{CO}(2-1)$ traces denser gas than $\mathrm{CO}(1-0)$. The combination of sensitivity to denser gas and the lack of sensitivity to diffuse extended structure is evident in a comparison of our map of the $\mathrm{CO}(2-1)$ integrated intensity with the map of $\mathrm{CO}(1-0)$ integrated intensity (Matsushita et al. 2005). The structure in the $\mathrm{CO}(1-0)$ integrated emission that defines the molecular wall of a superbubble in the nucleus of M82 (Matsushita et al. 2005) is not identifiable as such in our map of CO(2-1) integrated emission. The high velocities of the molecular gas defining the boundary of the superbubble are seen in our data, and these velocities are consistent with molecular gas in an expanding bubble (Matsushita et al. 2005) rather than molecular gas in a spiral arm (Shen \& Lo 1995). There is no report in the literature of the total emission in $\mathrm{CO}(2-1)$ that could be used to quantify how much of the large scale structure our interferometric observations are missing. 


\section{The Mass Spectrum of Clouds and Clusters}

\subsection{Details of the Observational Determination of the Mass Spectrum of the Clouds}

The CO observations are of course a projection of the emission on the plane of the sky and a determination of the three dimensional distribution of the molecular gas so that individual clouds can be identified is an interesting research topic itself (Williams, de Gues, \& Blitz 1994; Bensch, Stutzki, Ossenkopf 2001; Stutzki \& Gusten 1990). Here we rely on previous research and use the well defined algorithm clumpfind as detailed in Williams, de Gues, \& Blitz (1994). This algorithm uses contours of the CO emission to define the boundaries of individual clouds on the two dimensional map plane, and the gas velocities as determined by the spectral line information to define the boundaries on the third dimension. We used clumpfind to identify individual clouds to a threshold of twice the rms noise in a single channel as suggested by Williams, de Gues, \& Blitz (1994). Because the map of integrated intensity (figure 1) was also made with a threshold of $2 \sigma$, all the clouds seen in figure 1 represent at least one clump as determined by the clumpfind algorithm. A total of 303 discrete clouds were found by the algorithm.

Once the boundaries of the clouds have been identified, the determination of the enclosed mass, mostly $\mathrm{H}_{2}$, from the information provided by the $\mathrm{CO}$ tracer is another interesting research topic. Recent estimates of the conversion factor between the integrated emission of $\mathrm{CO}(1-0)$ and the column density of $\mathrm{H}_{2}$ in galaxies vary by a factor of 10 , and may depend on whether the galaxy is a starburst. Rosolowsky et al. (2003) estimate $2 \times 10^{20} \mathrm{H}_{2} \mathrm{~cm}^{-2}$ $\left(\mathrm{K} \mathrm{kms}^{-1}\right)^{-1}$ for M33, a relatively normal spiral. This conversion factor is the same as that estimated for our Galaxy (Strong et al. 1988). For starburst galaxies, Davies, Tacconi, \& Genzel (2004) estimate a factor of 0.4 to 0.8 times the Galactic value, while other studies find factors ranging from 0.2 to 0.1 (Wang et al. 2004; Wada \& Tomisaka 2005). Matsushita et al. (2000) suggest a conversion factor of $1.4 \pm 0.6 \times 10^{20}$. We adopt a value of $1.0 \times 10^{20}$ $\mathrm{H}_{2} \mathrm{~cm}^{-2}\left(\mathrm{~K} \mathrm{kms}^{-1}\right)^{-1}$.

The empirical conversion factors above are all determined for the integrated intensity of $\mathrm{CO}(1-0)$ whereas our observations are of $\mathrm{CO}(2-1)$. Because the conversion factor is essentially empirical, there is little theoretical guidance as to how one should modify this factor for the (2-1) line. Depending on the excitation temperature the population in the $(2-1)$ line may be different from that in the (1-0) line. Excitation temperatures can be estimated from observed ratios of certain molecular lines. Temperatures derived for the clouds in M82 are $11.5 K \pm 3.0$ from cyclopropenylidene in a $30^{\prime \prime}$ beam (Oike et al. 2004), $30 \mathrm{~K}$ from $\mathrm{NH}_{3}$ in a $40^{\prime \prime}$ beam (Mauersberger et al. 2004), and $50 \pm 20 \mathrm{~K}$ from $\mathrm{CO}$ in a $20^{\prime \prime}$ 
beam (Petitpas \& Wilson 2000). We can bracket the problem. For excitation temperatures between 5 and $50 \mathrm{~K}$, the line ratios in local thermodynamic equilibrium vary between 0.5 and 1.5. Thus over this range of temperature, a population ratio of unity should be within a factor of 2 and in agreement with a line ratio of 0.9 found empirically in a CO survey of nearby galaxies (Braine \& Combes 1992). The difference in brightness of the two lines owing to the frequency dependence of the source function will be accounted for in the conversion from $\mathrm{Jy}_{\text {beam }}{ }^{-1}$ to $\mathrm{K}$, measured to be $23 \mathrm{Jy} \mathrm{K}^{-1}$ in our calibration.

While a determination of the absolute cloud masses from the CO line emission is known to be uncertain (Wilson 1995; Israel 1997), the direct conversion still provides an indication of the masses. Furthermore, the relative masses, and therefore the slope of the mass spectrum, should be less affected by conversion uncertainties. The formal statistical uncertainty in the mass as derived from the propagation of the error in the measured flux in each pixel is relatively small compared to the uncertainty in the conversion factor. The average of the formal error of each of the clouds is $2 \%$, while the uncertainty in the conversion factor is on the order of at least a factor of a few.

The mass derived from the integrated intensity of CO may be compared to the virial mass derived from the length scale and the internal velocity dispersion. The use of the virial theorem in estimating the masses of molecular clouds has a long and successful history in studies of the ISM. However, recent theoretical work suggests that interstellar clouds may not be gravitationally bound entities in equilibrium, but rather transient structures in turbulent flows (Larson 1981; MacLow \& Klessen 2004; Elmegreen \& Scalo 2004). If so, the concept of virial equilibrium may not apply to interstellar clouds. However if the turbulence itself is driven by gravitational forces, that is if the velocities are primarily driven by the gravitational potential of the mass of the ISM, then there would be approximate equipartition of kinetic and potential energies within the flow Larson (1981). In this case, even though the clouds are not in virial equilibrium, the virial relationships should still apply. We use the equation for the virial mass, equation A3 in Williams, de Geus, \& Blitz (1994), that expresses the mass in quantities reported by the clumpfind algorithm, $M_{\text {vir }}=5 \Delta R \sigma_{v}^{2} / \alpha G$, where $\Delta R$ is the cloud radius, $\sigma_{v}$ is the line-of-sight internal velocity dispersion, and $\alpha$ is a geometrical factor equal to $5 / 3$ for a density profile scaling as $r^{-2}$. The radius derived from the clumpfind algorithm has been corrected for the spatial resolution following Equation A7 of Williams, de Geus, \& Blitz (1994). Clouds that have clump radii less than the beam size have been set to a size equivalent to a single pixel, or $0.2 \times 0.2$ square arcseconds. The data are not similarly corrected for the spectral resolution because the channel width of the data is the same width and function as the spectral resolution. Because of the finite spatial and spectral resolution, the masses of some of these clouds may be upper limits. The formal error on the virial mass, based on an assumed error in $\Delta R$ equal to the half-width at half maximum of 
the beam and an assumed error in $\sigma_{v}$ of half the channel width, is $100 \%$. The error in the virial mass is thus of the order of the uncertainty in the conversion mass.

Figure 2 shows that with the adopted CO-to- $\mathrm{H}_{2}$ mass conversion, the clouds in M82 follow the relationship of virial equilibrium. The lowest-mass clouds, below $10^{4} M_{\odot}$, appear on average to be slightly more massive than required for equilibrium. If one excludes the clouds with masses below $10^{4} M_{\odot}$, the fit is again indistiguishable from virial equilibrium. This result confirms, a posteriori, the adopted $\mathrm{CO}$-to- $\mathrm{H}_{2}$ conversion factor and suggests that the conversion factor in M82 differs from the standard Galactic value by a factor of 2 .

A histogram of cloud masses is shown in figure 3 , binned on a scale of $\Delta \log M=0.165$ that provides 20 bins of equal logarithmic size over the logarithmic mass range of the clouds from 3.4 to $6.7 \log \mathrm{M}_{\odot}$. Over this mass range, the mass spectrum can be reasonably described by a single power law, $\mathrm{d} N / \operatorname{d} \log M \propto-0.5 \pm 0.04$, that on a linear scale corresponds to $N(M) \propto M^{-1.5 \pm 0.04}$. The least squares fit to the slope includes all the clouds and assumes that the data $N(\log M)$ have equal weight.

The histogram includes all the clouds found by the clumpfind algorithm. Since the interferometer is only sensitive to emission on scales of $1^{\prime \prime}$ to $10^{\prime \prime}$, the mass spectrum at either end of the range of spatial scales 17 to $170 \mathrm{pc}$ is uncertain. Thus the completeness of the sample may be primarily dependent on the sensitivity to angular scale rather than, as in the optical case (Whitmore et al. 1999), on the confusion with the background emission. In interferometric observations the larger scale background emission is essentially invisible

At the lower end of the mass range, where the cloud sizes are below the resolution limit of the interferometer, the finite resolution of the interferometer causes both an increase in the apparent size of the cloud as well as an approximately offsetting decrease in measured antenna temperature. Thus these two errors may cancel resulting in higher uncertainty rather than a trend. Nonetheless, if one wishes to disregard the clouds at the lower end of the mass range, setting the lower mass limit to $10^{4} M_{\odot}$ results in a slope of $-1.6 \pm 0.06$ rather than $-1.5 \pm 0.04$. At the upper end of the mass range, the expected errors are somewhat different. The masses of the clouds at the upper end of the mass range may represent lower limits to true masses if the sizes of the clouds exceed the angular scale of $\sim 10^{\prime \prime}$ that is captured by the interferometer. There is no way of estimating the mass or structure of the clouds beyond the angular limit from the data set. However, this uncertainty affects only the most massive clouds. A fit to the histogram ignoring clouds with masses $>10^{6} M_{\odot}$ results in a slope of $-1.4 \pm 0.05$. The different slopes of the mass spectrum derived with the different mass limits suggests that the errors are dominated by uncertainties at either end of the mass range. Thus, the slope of the mass spectrum may be approximately estimated as $-1.5 \pm 0.1$. 


\subsection{Comparison of the Mass Spectrum of the Clouds and Clusters}

The mass spectrum of the molecular clouds in M82 over the mass range that we observe with the interferometer, $\sim 10^{3}-10^{7} \mathrm{M}_{\odot}$, is well approximated by a power law with a slope of -1.5 . The nominal mass spectrum of the SSCs in M82, calculated from continuum magnitudes measured by the HST, and taking into account an estimated extinction and age, but uncorrected for completeness, is peaked at a mass of about $10^{5} \mathrm{M}_{\odot}$ (Melo et al. 2005; de Grijs et al. 2001). Investigation of the completeness of HST observations of SSCs in the Antennae galaxies suggests that the peak in the distribution is due to the incomplete detection of faint clusters below the peak (Whitmore et al. 1999). Correction for the incompleteness results in a power law distribution for the luminosity. If the peak in the mass spectrum of the clusters in M82 is due to incompleteness, we may still derive the slope of the power law distribution by fitting the upper end of the cluster mass spectrum where the data are assumed to be complete. We derive a slope of -1.4 (figure 4 in our paper) from the masses of all the clusters listed in Melo et al. (2005) (tables $5-9$ ). (This power law distribution in the upper end of the mass range of the M82 clusters is similar to the power law mass spectra measured in other galaxies such as the Antennae and M51 (Zhang \& Fall 1999; Bik et al. 2003)). Thus the slopes of the mass spectra of the clouds and clusters in M82 are consistent with each other and consistent with the hypothesis that the mass scale of the molecular clouds determines the mass scale of the clusters. Furthermore, the slope of the mass spectrum of clouds in M82 is comparable to the slopes of the upper end of the mass spectra of old globular clusters in a number of galaxies (Harris \& Pudritz 1994) including M87 $(\alpha=-1.60)$, NGC1399 $(\alpha=-1.61)$, NGC4594 $(\alpha=-1.54)$, and NGC4636 $(\alpha=-1.90)$. If the lower end of the mass spectrum of the globular clusters can be assumed to be determined by the destruction

of low mass clusters, then the mass spectra of the clouds, SSCs and globular clusters are consistent with the hypothesis of a common origin for globular clusters and SSCs.

\section{Comparison with Indicators of Star Formation}

The relationship between the dense molecular clouds and star formation may be explored by comparing the $\mathrm{CO}$ emission with several indicators of star formation such as the SSCs themselves, radio point sources, diffuse radio free-free emission, and mid-IR emission. Each of these observables provides different information on the star formation activity because of their relationships to different emission mechanisms and because of the different extinction at the different wavelengths.

The SSCs, as identified by HST I-band and $\mathrm{H} \alpha$ emission (Melo et al. 2005), are seen to be spread throughout the nuclear region except at the locations of the brightest $\mathrm{CO}$ emission 
in the larger clouds (figure 5). Because dense molecular gas obscure the optical light from the SSCs, the inverse correlation between the SSCs and the CO emission does not necessarily imply a lack of clusters in the molecular clouds. But the presence of the clusters around the clouds certainly indicates recently completed star formation around the clouds. We know that the star formation is essentially finished in these optically visible clusters because if there were dense gas within the clusters available for further star formation, then this gas within the clusters would also obscure much of the optical emission from the clusters.

The centimeter-wavelength radio point sources (Rodríguez-Rico et al. 2004) are associated with supernova remnants and H II regions and the two may be distinguished by their radio spectral indices. In addition the HII regions may have detectable recombination line emission. Although the number of radio point sources and the number of bright clouds are both few, the radio point sources in figure 6 appear clustered around the edges of the clouds, but absent in the centers of the brightest $\mathrm{CO}$ clouds with the exception of the one large cloud at $9 \mathrm{~h} 55 \mathrm{~m} 51.4 \mathrm{~s}+69 \mathrm{~d} 40$ ' 44 " (marked A on figure 1) that is coincident with several HII regions. If this inverse correlation is significant, then because the centimeter emission is little affected by extinction, the absence of supernovae and HII regions within the molecular clouds indicates a lower rate of star formation in the centers of most of the clouds than on their periphery.

If this peripheral star formation is currently ongoing, it should be occurring in molecular gas. The lack of detection in our $\mathrm{CO}$ observations indicates that this gas mst have a characteristic scale exceeding 10" that is beyond the range of spatial scales detectable by the interferometer. Alternatively, the star formation may have consumed and dispersed the molecular gas around the dense clouds. In this case, the star formation must have just finished.

Mid-infrared emission indicates the presence of warm $(\mathrm{T} z 100 \mathrm{~K})$ dust and is often associated with massive star formation. A comparison of the mid-IR emission (Lipscy \& Plavchan 2004) with the CO emission shows an association, but again a lack of detailed correspondence (figure 7) with the exception of the large cloud at 9 h $55 \mathrm{~m} 51.4 \mathrm{~s}+69 \mathrm{~d} 40$ ' 44 " (marked A on figure 1). and a small cloud to the west (right) at $9 \mathrm{~h} 55 \mathrm{~m} 51.4 \mathrm{~s}+69$ d 40 ' 44 " (marked B on figure 1). The mid-IR emission within the large cloud " A" is not coincident with the peaks of the CO emission but forms an arc just to the south and west around the brightest CO peak. Since the mid-IR emission is little affected by extinction, the weak mid-IR emission in the centers of most of the clouds again suggests less star formation activity in the centers of the most of the $\mathrm{CO}$ clouds.

Matsushita et al. (2005) suggest that the diffuse $100 \mathrm{GHz}$ continuum emission in M82 is a tracer of star formation activity because it is dominated by free-free emission from gas 
that has been ionized by supernovae-driven shocks and the radiation from massive stars. The comparison (figure 6) between the continuum and the $\mathrm{CO}$ emission indicates star formation in the same two regions as indicated by the mid-IR although the $100 \mathrm{GHz}$ continuum observations do not have the angular resolution to determine the location of the star formation within the largest cloud. Similar to the mid-IR, the $100 \mathrm{GHz}$ continuum indicates star formation around all the molecular clouds, but no increase at the locations of most of the clouds. Because the centimeter radio emission is little affected by extinction, the lack of continuum emission in the centers of most of the clouds indicates no increase in star formation within these clouds.

In summary, the comparisons of the $\mathrm{CO}$ with several tracers of star formation activity fail to find evidence for a correspondence between current star formation and the column density of CO. Only one of the large clouds shows star formation activity in its interior and then only on one side, just off the center. Only one smaller cloud shows evidence of interior star formation aligned with the CO peak. Instead current star formation generally appears around the edges of most of the largest clouds. The several comparisons can be rationalized into a hypothesis of inwardly progressing star formation. The distribution of SSCs around the dense molecular clouds suggests that star formation has occurred in the surrounding area over the past $25 \mathrm{Myr}$ with about half the clusters formed in the last 6 Myr (Melo et al. 2005). The distribution of supernovae and HII regions suggests that star formation is occurring on the periphery of the giant molecular clouds at the current time. If the centers of molecular clouds are sites of future star formation, the observations are consistent with star formation propagating inward through the molecular clouds from the edge toward the center. This inwardly propagating star formation is on the scale of individual clouds $(\$ 50$ pc) and is a smaller scale than that of the outwardly propagating star formation (500 pc) suggested by Satyapal et al. (1997). The two hypotheses are quite consistent with each other if an earlier generation of star formation triggers further star formation by compression of the molecular clouds.

\section{Triggering the star formation}

The mid-IR and $100 \mathrm{GHz}$ radio continuum identify two regions with the most active star formation, the large cloud at 9 h 55 m $51.4 \mathrm{~s}+69 \mathrm{~d} 40$ ' 44 " (marked A on figure 1) and the small cloud to the west (right) at $9 \mathrm{~h} 55 \mathrm{~m} 51.4 \mathrm{~s}+69 \mathrm{~d} 40$ ' 44 " (marked B on figure 1). Why is star formation most active in these two clouds and not elsewhere? The observational evidence is consistent with the hypothesis that the star formation is triggered by a compressive shock driven into the molecular clouds. This is determined from the data 
as follows. A plot of the intensity weighted average velocity, $\bar{v}=\langle I v>/<I>$ shows the velocity gradients in the clouds by the change in $\bar{v}$ (figure 7 ). The velocity gradients are strongest where the mid-IR emission is strongest and the comparison is precise in detail. The smaller cloud with the brightest mid-IR emission has a circular pattern of $\bar{v}$ consistent with radial flow centered on the location of the strongest mid-IR emission. A spectrum (figure 8) shows an inverse P-Cygni spectral line profile with red-shifted absorption and blue-shifted emission. This profile arises from the mixture of ionized gas (continuum emission figure 6) and the molecular gas within the cloud. The red-shifted absorption places the gas moving away from the observer unambiguously on the near side of the cloud. Therefore this gas is moving toward the center of the cloud. Similarly the blue-shifted gas seen in emission must be from behind the ionized gas, on the far side of the cloud moving toward the observer and toward the center of the cloud. The inward velocity is about $35 \mathrm{kms}^{-1}$ as measured by the half the difference in the line center velocities of emission and absorption. The velocity field thus shows that this molecular cloud is radially contracting at highly supersonic velocities consistent with its being crushed by a sudden increase in external pressure. The spherical pattern of the flow is consistent with an isotropic external pressure as would be provided by hot ionized gas.

A comparison of the velocity field in the larger cloud (marked A on figure 1) to the east (left) with the mid-IR emission again shows that where the gas is in supersonic compression, the star formation activity (mid-IR emission) is the highest. The ridge of mid-IR emission is aligned with the lateral velocity gradient seen in the change in $\bar{v}$ in figure 7 . Individual spectra in this cloud shows why star formation is active on the west (right) side of the velocity gradient and the cloud and not on the left. Figure 9 is a map of spectra across the cloud. The spectra from the west (right) side of the cloud show inverse P-Cygni profiles with red-shifted absorption indicating compression, while the spectra from the east (left) side show classic P-Cygni profiles with blue-shifted absorption indicating expansion. The inward velocities are similar to those in the smaller cloud, again 20 to $30 \mathrm{~km}^{-1}$. Thus the velocity field in this cloud shows that where the cloud is in compression, star formation is active, and where the cloud is expanding star formation is quiet. The column density of the molecular gas, proportional to the integrated intensity of CO, is similar on both sides of the cloud. Thus the compression due to the velocity field and not the density alone is the difference that is related to the difference in star formation activity.

The third region of weaker star formation activity further east (left) at 9 h $55 \mathrm{~m} 53 \mathrm{~s}+69$ d 40m $47 \mathrm{~s}$ (marked $\mathrm{C}$ on figure 1), shown by the mid-IR emission on figure 7. also occurs where the clouds have an internal velocity gradient. Comparison with the CO integrated intensity figure 1 , indicates that the very strong change in $\bar{v}$ at this position is from the overlap of two different clouds. The star formation is associated with the velocity gradient 
within each cloud. This gradient is apparent in the much more subtle change in color across each individual cloud.

Why the most active star formation is occurring in two particular clouds and not the other similar clouds may or may not be a significant question. If star formation is triggered and sequential, then these clouds may be the current location of the progression of star formation. The other clouds may be in turn be triggered into compression in the near future. The alternative is that the location is in some way special in relation to the galaxy, apart from the current starburst.

The observation of the velocities of the molecular clouds provides unambiguous evidence for the causal relationship of the compression of giant molecular clouds and star formation. There is no ambiguity as to whether the star formation is related to the compression or whether star formation is proceeding inside the clouds of its own course for example because of high density. In the case of M82, only those clouds that are in compression show active star formation despite the similarities in density of the largest clouds. In one cloud, star formation is occurring in the side under compression and not in the side that is expanding. The two

sides of the clouds are otherwise observationally indistinguishable. Thus the observations imply that star formation in starbursts is essentially a triggered phenomenon.

\section{Theoretical Considerations and Hypotheses}

The observations suggest that star formation in starbursts is triggered by the collapse of giant molecular clouds driven by a sudden increase in external pressure. The ionized gas seen around the molecular clouds is one possible source of this pressure as is radiation pressure from a previous generation of stars. The observations can be used to understand the origin of this driving pressure and its more detailed effects on the giant molecular clouds by comparing estimates of the physical conditions in M82 against the conditions predicted by different hypotheses about starbursts and cluster formation. The results of this comparison of theory against observation allow us to describe a hypothetical general scenario for the starburst process in galaxies similar to M82.

\subsection{The driving pressure for the collapse of giant molecular clouds}

The ionization may have been caused by strong shocks resulting from cloud collisions or the pressure of supernovae explosions in a previous generation of starbursts. A scenario for the ionization of clouds by collisions and the subsequent compression of giant molecular has 
been outlined by Jog \& Solomon (1994) (JS). They supposed that the collision of HI clouds in interacting galaxies would lead to their ionization. Because of their smaller filling factor, the giant molecular clouds would not collide directly, and as a result they would not be ionized by collision, but rather would be surrounded by hot ionized gas at a pressure substantially higher than provided by the HI clouds in their previous atomic state. Alternatively, Matsushita et al. (2005) attribute the ionization of the gas to shocks from a previous starburst episode that they identify with observed $2.2 \mu \mathrm{m}$ emission. We will examine these two hypotheses with respect to the several observations of M82.

In their analysis of cloud collisions JS supposed a relative velocity of $300 \mathrm{kms}^{-1}$, a typical relative velocity of two galaxies. The galaxy M82 is not obviously in a direct collision but shows evidence of gravitational interaction with the neighboring galaxy M81 (Yun et al. 1993). The interaction may still result in the collision of clouds within M82 by the distortion of the rotational equilibrium that existed in M82 prior to its close encounter with M81. Since the rotational velocity in a typical galactic disk is about $200 \mathrm{~km}^{-1}$, and since our CO observations show a velocity gradient of about $200 \mathrm{kms}^{-1}$ across the 15 " (261 pc) in our map. we might expect a relative velocity of this order, similar to the relative velocity chosen by JS.

In the following analysis, we will assume that shocks initially heat the gas to a high temperature and that the post-shock gas is subsequently rapidly cooled by radiation. In discussing the energetics of shocked gas, the simple adiabatic and isothermal approximations are useful even though we do not expect the shock to conform to either of these extremes. In an adiabatic shock a substantial portion of the kinetic energy of the colliding clouds is converted into thermal energy of gas. In an isothermal shock, all this thermal energy is assumed to be immediately radiated away so that the gas returns to its pre-shock temperature but at a higher density. We will use the adiabatic approximation to describe the post-shock gas in its brief hot phase and the isothermal approximation to describe the post-shock gas after it has cooled.

We estimate the temperature and density of the post-shock gas before it has had time to cool with the adiabatic approximation (Spitzer 1978; equations 10-22 and 10-23). In the limit of high Mach number, the compression of an adiabatic shock will be

$$
\rho_{2} / \rho_{1}=\gamma+1 / \gamma-1
$$

where $\rho$ is the density and the subscripts 1 and 2 indicate the pre and post-shock gas, and $\gamma$ is the adiabatic index. The increase in energy density or pressure, $P_{2}$, of the gas following the passage of a shock with velocity, $u_{1}$ is,

$$
P_{2}=2 \rho_{1} u_{1}^{2} / \gamma+1
$$


For an initial density in HI of $20 \mathrm{~cm}^{-3}$ (JS) and shock velocity equal to the relative velocity of $200 \mathrm{kms}^{-1}$, the post shock density and temperature will be $80 \mathrm{~cm}^{-3}$ and $10^{6} \mathrm{~K}$ for a gas with an adiabatic index of $\gamma=5 / 3$. This predicted temperature is the same as the $10^{6} \mathrm{~K}$ determined observationally from X-ray observations (Griffiths et al. 2000). The density of the ionized gas may be estimated from the $100 \mathrm{GHz}$ continuum observations of Matsushita et al. (2005). Following Mezger and Henderson (1967),

$$
\left(n_{e} / \mathrm{cm}^{3}\right)^{2}=8 \times 10^{7}\left(S_{\nu} / m J y / \text { beam }\right)\left(T_{e} / 10^{4}\right)^{0.35}(D / k p c)^{-1}(\nu / G H z)^{0.1}\left(\theta_{x} \theta_{y} \theta_{z} / \operatorname{arcsec}^{3}\right)^{-1}
$$

where $n_{e}$ is the electron density, $S_{\nu}$, is the flux density, $T_{e}$ is the electron temperature, $D$ is the distance, and $\theta$ the dimensions of the emitting region. The $100 \mathrm{GHz}$ flux away from the positions of peak emission is about $5 \mathrm{mJy} /$ beam (Matusushita et al. 2005). If the path length is $100 \mathrm{pc}$, the approximate width of the ionized and X-ray emitting gas, then $\theta_{z} \sim 6$ ". The width in the map plane $\theta_{x} \times \theta_{y} \sim 5 \times 4$ ", twice the FWHM. Then the number density would be $90 \mathrm{~cm}^{-3}$ comparable to the theoretical estimate.

The shocked gas will cool rapidly,

$$
t_{\text {cool }}=(3 / 2) n k T / \Lambda
$$

where the numerator is the energy density of the gas and $\Lambda$ is the cooling rate (McKee \& Cowie 1977).

$$
\Lambda=6.2 \times 10^{-19} T^{-0.6} n^{2}
$$

The cooling timescale of the shocked gas is about $10^{3}$ years. Thus in the absence of a continuous source of energy to maintain the high temperature, one would expect to gas to cool rapidly and we should observe HI clouds rather than ionized clouds. If we approximate the properties of the cooled post-shock gas by the isothermal approximation, the post-shock HI clouds should have a density equal to the initial density times the Mach number squared. If the pre-shock HI had an effective sound speed equal to a typical velocity dispersion of 1 $\mathrm{kms}^{-1}$ (Spitzer 1978), then the Mach number would be 200, and the post-shocked HI gas would be compressed to an enormously high value. Thus although a single episode of cloud collisions would provide conditions similar to those observed, unless we are observing M82 at the precise moment of the collision, it remains to be understood how the energy input from collisions could be maintained.

Let us examine the hypothesis that the ionizing energy is provided by a previous starburst. Matsushita et al. (2000) identify a $2.2 \mu \mathrm{m}$ peak, which is located at a position between the two regions of most active, star formation as emission from an embedded massive star cluster. They estimate a mass in stars of $2 \times 10^{6} \mathrm{M}_{\odot}$ and 4000 supernovae explosions over the past Myr for a rate of about 4 supernovae explosions in a cooling time of $1000 \mathrm{yr}$. The kinetic 
energy release per supernova (type I and II) is $4 \times 10^{50}$ ergs (Spitzer 1978, pg 231; Blair \& Kirshner 1985) If this energy goes into a volume of $100 \mathrm{pc}^{3}$, then the energy density supplied in a cooling time is $4 \times 10^{7} \mathrm{~K} \mathrm{~cm}^{-3}$. This is close to the estimated energy density $\left(10^{8} \mathrm{~K}\right.$ $\mathrm{cm}^{-3}$ ) required to maintain the temperature of the ionized gas at $10^{6} \mathrm{~K}$. Thus the energy input from a single star cluster is sufficient to maintain the ionized gas at high temperature.

The surface density of SSCs in areas around the molecular clouds is about 20 per $100^{2}$ $\mathrm{pc}^{2}$. Thus in the area of the ionized gas, there could be more than one cluster, but hidden behind the extinction of the molecular gas. Thus the energy available from supernovae explosions in several clusters is more than sufficient to maintain the ionization of the ISM.

\subsection{The effect of the pressure on the giant molecular clouds}

The excess pressure of this hot ionized gas will drive a shock into the neighboring cold molecular clouds at a speed set by the momentum jump condition,

$$
v_{s}=\left(\rho_{\text {ion }} / \rho_{\text {mol }}\right)^{1 / 2} c_{s}
$$

Rounding off the estimates in the previous section we may assume that the energy density of the ionized gas $10^{8} \mathrm{~K} \mathrm{~cm}^{-3}$, the temperature is $10^{6} \mathrm{~K}$ and the density $100 \mathrm{~cm}^{-3}$. The sound speed in the $10^{6} \mathrm{~K}$ ionized gas is $90 \mathrm{kms}^{-1}$. With an initial density of $100 \mathrm{~cm}^{-3}$ for the uncompressed molecular gas (JS), the shock speed in the molecular gas will be $60 \mathrm{kms}^{-1}$. The post-shock gas will be accelerated to some significant fraction of this shock speed, and the observed inward velocities of $35 \mathrm{kms}^{-1}$ are thus consistent with the estimated shock speed.

In the brief adiabatic phase, the shock will heat the gas to a temperature of $2 \times 10^{5}$ with a density of $400 \mathrm{~cm}^{-3}$ (equations 1 and 2) assuming a molecular weight of 2.33 . The gas will cool rapidly, molecules will reform, and the increase in density due to the shock will be,

$$
n_{\text {final }} / n_{G M C}=M^{2}
$$

where $M=15$ is the Mach number of the shock assuming $v_{s}=60 \mathrm{kms}^{-1}$ and the effective sound speed in the molecular gas is equal to the velocity dispersion of $4 \mathrm{kms}^{-1}$, appropriate according to the size linewidth relationship (Larson 1981), for a typical molecular cloud of 25 pc. For the assumed initial molecular density of $100 \mathrm{~cm}^{-3}$, the post-shock density will be $2 \times 10^{4} \mathrm{~cm}^{-3}$, within a factor of a few the same as the average molecular density of the clouds in our sample $\bar{n}=4500 \mathrm{~cm}^{-3}$ as determined from the CO observations. Because the distribution of cloud sizes is a scale free power law, the averages reflect only the properties of the clouds covered by the range of our sample. 
The first stars should appear in the post-shock layer in about a free-fall time,

$$
t_{f f}=((32 / 3 \pi) G \rho)^{-1 / 2}
$$

or $2 \times 10^{5}$ yrs for a density of $2 \times 10^{4} \mathrm{~cm}^{-3}$. At this time the layer will be $15 \mathrm{pc}$ thick if the shock speed is $60 \mathrm{kms}^{-1}$. For our typical molecular cloud with a radius of $25 \mathrm{pc}$ and number density of $100 \mathrm{~cm}^{-3}$, the shocked layer will be in the form of a dense shell at the periphery of the cloud. The mass of the shell will be $3 \times 10^{5} \mathrm{M}_{\odot}$. The star formation rate will be

$$
S F R=M_{\text {stars }} / t_{f f}
$$

If we assume a star formation efficiency of $50 \%$, the minimum required to form a bound star cluster, the star formation rate in the compressed shell of one cloud will be $0.6 \mathrm{M}_{\odot} \mathrm{yr}^{-1}$. This is also approximately the star formation rate for the whole cloud if the shock progresses to the center of the cloud.

\subsection{Star formation by radiative compression}

Suppose the pressure that is driving the shock is reduced if the hot ionized gas surrounding the molecular cloud cools or if its density is reduced by outflow in a galactic wind. Star formation may continue nonetheless because the radiation pressure from the first stars that are formed in the post-shock gas will be sufficient to continue the compression. If the first star formation in an interacting galaxy is initiated by the collision and ionization of HI clouds as suggested by JS, then the radiation pressure from the first stars formed may allow star formation to continue through a cloud despite the short time scale for the collisional ionization discussed above and in JS.

In the example in the section above, the total luminosity of the stars in the compressed layer will be (JS; Scoville \& Young 1983)

$$
L_{t o t}=1.3 \times 10^{10}\left(L_{\odot} y r / M_{\odot}\right) S F R
$$

or $8 \times 10^{9} \mathrm{~L}_{\odot}$. This luminosity will generate an inward radiation pressure of $4.5 \times 10^{8} \mathrm{~K}$ $\mathrm{cm}^{-3}$, assuming the pressure is given by,

$$
P=(1 / 2) L /\left(4 \pi r^{2} c\right)
$$

with $c$ the speed of light. Thus the radiation pressure from the first wave of star formation is of the order of the pressure of the ionized gas that began the compression of the molecular cloud. Therefore the star formation will now be inwardly self-propagating. The inward 
speed of the wave of star formation will be equivalent to the initial shock speed owing to the similarity in the pressure of the ionized gas and the radiation pressure of the shell of star formation. Thus once star formation is triggered by compression, the star formation will move inward at supersonic velocities even if the external pressure is rapidly reduced. The possibility of self-propagating star formation triggered by radiation pressure is a possible means around the problem discussed in JS of the short time scale for compression due to cloud collisions.

\subsection{Fragmentation by thermal and gravitational instabilities}

If a high Mach number shock is driven into a molecular cloud so that the cloud is temporarily ionized, then upon cooling, the gas may fragment by thermal and gravitational instabilities. Because the temperature enters into the equation for the cooling rate (equation 5) as a negative power, the gas is thermally unstable under both isobaric and isochoric conditions (equation 4, Field (1965)). Fragmentation by the thermal instability is especially interesting because it allows for a fragmentation on time and length scales that would shorter than those expected from gravitational collapse.

The thermal instability will be most effective in fragmenting the gas if the timescale of the thermal instability is comparable to the dynamical time scale of a fragment. The growth rate of the thermal instability is roughly equal to the cooling rate of the gas. If the post-shock gas is heated to $2 \times 10^{5} \mathrm{~K}$ and compressed to $400 \mathrm{~cm}^{-3}$, then the cooling time (equation 4 ) is only $10 \mathrm{yr}$. The dynamical time scale is given by a length divided by the sound speed. The length scale of a density perturbation that would give rise to a condensation of a solar mass would be about $0.3 \mathrm{pc}$, as given by,

$$
\lambda=(3 M / 4 \pi \rho)^{1 / 3}
$$

Assuming a sound speed of $100 \mathrm{kms}^{-1}$ appropriate for gas at $2 \times 10^{5} \mathrm{~K}$, the dynamical time scale for a perturbation of this size $\lambda / c_{s} \sim 3000 \mathrm{yrs}$. The disparity in time scales implies that the gas will cool under isochoric conditions. Thus the thermal instability as described by linear perturbation analysis will be unable to compress the gas in the colder regions before all the gas has cooled and the thermal instability ceases. However, non-linear effects may allow the thermal instability to play a role in fragmentation (Murray \& Lin 1989; Murray \& Lin 1991; Inutsuka \& Koyama 2004; Baek et al. 2005). Fragmentation by non-linear effects must be investigated by numerical methods beyond the scope of this paper.

Although the gravitational or Jeans instability in purely spherical geometry is unable to fragment a cloud, if there are pre-existing density perturbations in the cloud, the passage 
of the shock and subsequent compression of the gas may make some of these perturbations unstable to gravitational collapse. Once the post-shock gas has cooled, it will be significantly denser, about $2 \times 10^{4} \mathrm{~cm}^{-3}$, than in the pre-shock state, $100 \mathrm{~cm}^{-3}$. At this density, the size of a solar mass perturbation will be $\lambda \sim 0.07\left(\mathrm{M} / \mathrm{M}_{\odot}\right)^{-1 / 3}$ pc. The escape speed at the boundary of the perturbation will be, $v_{\mathrm{esc}}=(G M / \lambda)^{1 / 2}$ or $0.25\left(\mathrm{M} / \mathrm{M}_{\odot}\right)^{1 / 3} \mathrm{kms}^{-1}$. The perturbation will be unstable by the Jeans criterion if this escape speed exceeds the sound speed or effective sound speed of the molecular gas. What affect the shock will have on the velocity dispersion of the molecular gas is not clear. If the internal velocity dispersion of the post-shock gas is the same, $4 \mathrm{kms}^{-1}$, as in the pre-shock state, then a condensation of a few $100 \mathrm{M}_{\odot}$ will be unstable, and the compression of the cloud reduces the Jeans mass to at least 100 times smaller than in the pre-shock cloud where the Jeans mass was approximately the size of the entire pre-shock cloud.

The assumption that the sound speed of the cloud remains the same in the compressed cloud as in the original cloud results in a higher Jeans mass than in typical clouds because the compressed cloud has an internal velocity dispersion of the much larger pre-shock cloud and the compressed cloud then no longer follows the standard size-line width relation (Larson 1981). If the effective sound speed is reduced on passage through the shock then the Jeans mass will be lower than $100 \mathrm{M}_{\odot}$.

The process of fragmentation has interesting implications for properties of stars within clusters. For example, if fragmentation were to take place very rapidly as might be achieved if fragmentation were accelerated by the thermal instability, then the stars within the cluster might more likely have the same metallicity because they would be formed at the same time from the same molecular gas. If fragmentation takes place gravitationally, then if the Jeans mass of the compressed cloud were higher than in typical molecular clouds, the initial mass function (IMF) might be different in an SSC than in a typical open cluster in our galaxy.

\subsection{The dominant mode of star formation in starbursts}

Are all stars in a starburst formed in clusters? The star formation rate proceeds as the 1.5 power of the surface density according to the Schmidt law or according to simple scaling arguments, as the volume density divided by the free-fall time, $\rho / t_{f f}$. If the shock compression of the molecular gas is a factor of about 100, then the star formation rate in a shock compressed cloud will be 1000 times faster than in a normal giant molecular cloud. Thus most of the stars formed in a starburst will form in clusters.

Because the cloud mass spectrum is a power law extending at least to clouds of a few 
thousand $\mathrm{M}_{\odot}$, we would expect the compression of many small clouds and their transformation into clusters. These small clusters with their relatively weak gravitational binding energy might not survive very long against disruptive tidal forces in the crowded star burst environment. The dispersal of these cluster would liberate stars to the field. However with a power law mass spectrum most of the mass is in the largest clouds, and most of the stars in the starburst would remain in massive clusters.

\subsection{A universal process for the formation of open and globular clusters}

A fundamental question about the SSCs is whether the formation of these gravitationally bound massive clusters is as suggested by the hypothesis of shock compressed giant molecular clouds fundamentally different than the processes that we see operating in our own Galaxy, or whether a bound massive star cluster could be formed simply by a scaled up version of normal star formation. In cluster formation in our Galaxy, with a few possible exceptions (e.g. Clark et al. 2005), the formation of the first few massive OB stars tends to disperse the gas of the host giant molecular cloud resulting in a low efficiency for star formation and leaving insufficient stellar mass for the cluster to remain gravitationally bound. However, in the higher density environment of a starburst, because the star formation rate scales with the gas density as the $\sim 1.5$ power according to the Schmidt law (Schmidt 1959; Kennicutt 1998), perhaps the rate of star formation might be high enough to transform most of the cloud into stars before the dispersal of the molecular gas. Elmegreen \& Efremov (1997) (EE) have described just such a model for cluster formation with the additional hypothesis that the high pressure environment will also result in a lower dispersal rate that improves the efficiency. The lower rate of dispersal results from the higher gravitational binding energy of the clouds that in turn derives from the assumption of virial equilibrium in a high

pressure environment. A cloud in equilibrium in a high pressure environment must have a higher internal energy $\rho c_{s}^{2}$. To maintain virial equilibrium, the cloud must also have a higher gravitational binding energy that should result in a lower rate of dispersal.

In this model, the gas in a cloud is diminished by two processes: transformation to stars and dispersal by the energy of the newly formed stars. Star formation proceeds until there is no more gas in the cloud. The efficiency of star formation then depends on the relative rates of star formation and dispersal. Following EE, if the rate of star formation is proportional to some power of the density and the rate of dispersal is proportional to the stellar luminosity and inversely proportional to the gravitational binding energy of the cloud, then high density clouds will evidently form stars more efficiently. By scaling the rates of star formation and efficiency from those in the solar neigborhood, EE are able to show that a 
cloud in an environment with a pressure 1000 times higher, a cloud of $10^{4} \mathrm{M}_{\odot}$ is able to form stars at an efficiency of $50 \%$. This efficiency of course depends on the cloud beginning its star formation and dispersal at its assumed density and pressure. In other words, in a high density, high pressure environment, either the cloud is formed at that density and pressure, or star formation must be delayed until the cloud reaches a threshold density and pressure.

However, if the cloud is compressed from a lower density, then the star formation efficiency will be lower. For example, the rate of change of the mass of the gas in a cluster is given in EE by their equation (3),

$$
d M_{c} / d t=d M_{s} / d t-A L / c_{t}^{2}
$$

where $M_{c}$ is the mass of gas, $d M_{s} / d t$ is the star formation rate, and the last term is the rate of dispersal proportional to the total luminosity of stars in the cluster and inversely proportional to the internal turbulent velocity dispersion, $c_{t}$. If however, a low density cloud that is allowed to form stars, is brought to a higher pressure, always assuming virial equilibrium, then the equation for the rate of change of the mass of the gas in the cloud will be,

$$
d M_{c} / d t=d M_{s} / d t(1-\alpha t)\left(1-A L / c_{t}^{2}\right)(1-\alpha t)^{-1 / 4}
$$

where $\alpha$ is the rate of increase of the pressure, the star formation rate scales directly with the pressure, and the dispersal rate scales as $P^{-1 / 4}$. The latter two scalings are derived as in EE from virial equilibrium and the Schmidt law, $d M_{s} / d t \propto \rho^{1.5} \propto\left(P^{3 / 4}\right)^{1.4} \sim P$ and $c_{t}^{2} \propto M^{1 / 2} P^{1 / 4}$. The difference in the result of the two equations is plotted in figure 10 . The top line shows the efficiency for clouds of different masses that start forming stars at a pressure of 160 times that in the solar neighborhood. The lower dashed line shows the efficiencies for clouds that are brought up to this pressure over a crossing time and are allowed to form stars along the way as the pressure increases. Since these latter clouds spend some time at lower pressures, even the most massive clouds never reach an efficiency of $50 \%$ and will therefore never form a massive gravitationally bound cluster.

The pressure increase of 160 was chosen to match the conditions in M82. The ratio of the average density of the M82 molecular clouds to those typical in our Galaxy is about 45 . Since in this theory the density scales as the $3 / 4$ power of the pressure, the observed molecular density in our M82 clouds would correspond to an increase in pressure by a factor of about 160. At this overpressure, clouds of $10^{5} \mathrm{M}_{\odot}$ would have an efficiency of about $40 \%$ for the static case and $20 \%$ for the case of slowly increasing pressures. Thus star clusters produced in clouds of mass less than $10^{5} \mathrm{M}_{\odot}$ would be less likely to be gravitationally bound, and most would begin to disperse within a crossing time. Our sample of clouds shows a power law mass spectrum from $10^{6}$ down to below $10^{4} \mathrm{M}_{\odot}$. For a sample of star clusters with ages greater than a crossing time, this theory would predict a power law mass spectrum of slope -1.5 for 
star clusters with masses greater than the minimum mass required for gravitational binding, about $10^{6} \mathrm{M}_{\odot}$ and a declining cluster mass spectrum for masses below this value. Thus the cluster mass spectrum would be peaked at $10^{6} \mathrm{M}_{\odot}$ for the case of a static overpressure, and few bound clusters would be expected for the case of increasing pressure.

We can estimate the dispersal time of the stars in a cluster from its velocity dispersion and radius of a cluster. Observations of a few clusters indicate velocities of 10 to $15 \mathrm{kms}^{-1}$ for clusters with radii of a 1 and 2 pc and masses of $10^{5} \mathrm{M}_{\odot}$ (Ho and Filippenko 1996) The implied crossing and dispersal times are then about $10^{5}$ years. The stellar clusters in the nearby region of M82 known as B1 and B2 (deGrijs et al. 2000) have ages greater than $10^{8}$ yr and show equal numbers of SSCs detected above and below $10^{5} \mathrm{M}_{\odot}$. If the clusters are unbound below a mass of $10^{6} \mathrm{M}_{\odot}$, we would expect very few unbound clusters to survive for the age of the B1 and B2 regions which is more than 1000 crossing times. If the observed mass distribution were corrected for completeness (Whitmore et al. 1999), the number of low mass clusters in M82 B1 and B2 would be higher implying the formation of many bound clusters below the mass limit predicted by the theory of universal cluster formation. Thus the observations are inconsistent with the hypothesis of cluster formation by conventional (Galactic) star formation in a high pressure environment.

This result bears on the hypothesis of super-giant molecular clouds (Harris \& Pudritz 1994; Wilson et al. 2003). If the hypothesis of universal cluster formation in a high pressure environment is not satisfactory, then there is no motivation for supposing the existence of super-giant molecular clouds to provide the high pressure around internal giant-molecularcloud-sized clumps that will transform into massive star clusters. Furthermore, we do not detect super-giant molecular clouds in our observations. The largest cloud detected in our sample is less than $10^{7} \mathrm{M}_{\odot}$. Our observations are sensitive to structure as large as about 170 pc. A cloud of this dimension with density, $4500 \mathrm{~cm}^{-3}$ equal to the average density of clouds in our sample, would have a mass of $10^{8} \mathrm{M}_{\odot}$. Thus we should be able to detect clouds up to $10^{8} \mathrm{M}_{\odot}$, but perhaps not larger. Thus there is no motivation or evidence for super-giant molecular clouds in M82.

The hypothesis that individual SSC's are formed from individual giant molecular clouds is consistent with the observational finding that SSC's are found in complexes or clusters of SSC's (Whitmore et al. 1999; Melo et al. 2005). The implication then is that the individual molecular clouds were found in complexes prior to compression. This implied structure of the ISM is consistent with a number of theoretical conceptions about the ISM, for example the idea that the clouds are structures in a hierarchical turbulent cascade, or that the structure of the ISM has similarities with a fractal structure. Both turbulent and fractal structures are scale-free, but would appear clustered when not completely resolved by observations. 
Thus a complex of normal giant molecular clouds that would be transformed into a complex of SSC's could appear as a super giant molecular cloud when viewed with limited angular resolution.

The difference between the hypothesis of cluster formation within super giant molecular clouds and the hypothesis of cluster formation through shock compression as suggested by our observations is not so much a difference in the scale of the clouds but of the process of compression. If the clouds are cores within a larger super giant molecular cloud and compressed by a surrounding molecular cloud, then these cloud-cores would be compressed by molecular gas. In the alternative hypothesis of shock compression by the pressure of ionized gas as proposed for M82, the clouds are compressed by surrounding ionized gas. Secondly, although the hypothesis of formation within super giant molecular clouds does not specifically propose a time scale, if one wishes the process to remain consistent with a universal process of cluster formation, then the compression of a cloud-core within the super giant molecular cloud should not take places on a time scale so much shorter than the crossing time of the core, that a high velocity shock develops within the core. Star formation by shock compression is not the way star formation proceeds at lower pressures in our own Galaxy. Thus in the process of universal cluster formation, there is an implied minimum time scale of about a crossing time. In contrast, the time scale for cluster formation by shock driven compression could be much shorter, for example, the shock crossing time, or the free-fall time of the Jeans mass scale fragments discussed in the section on fragmentation.

\section{Conclusions}

We have resolved the ISM in the M82 starburst into 300 molecular clouds. The mass spectrum has a slope of -1.5 consistent with the unevolved upper end of the mass spectrum of super star clusters in M82 and of globular clusters other galaxies. The internal velocities of the giant molecular clouds with the most active star formation indicate massive compression on the scale of the entire cloud. Simple theoretical considerations suggest that the star formation is triggered by the compression of a shock driven into the molecular clouds by the pressure of the surrounding ionized gas. The wave of star formation may be self-propagating because the radiation pressure from the first stars formed is sufficient to continue the compression of the molecular cloud. This mode of star formation appears quite different from normal star formation in our own Galaxy, and in particular star formation in starbursts does not appear to be a scaled up version of star formation as we see in open clusters in our own Galaxy. 
The authors thank George Field and Doug Lin for their help in exploring the interesting possibility of the thermal instability. L. C. H. acknowledges financial support from the Carnegie Institution of Washington and by NASA grants from the Space Telescope Science Institute (operated by AURA, Inc., under NASA contract NAS5-26555).

\section{REFERENCES}

Aguilar, L., Hut, P., \& Ostriker J., 1988, ApJ, 335, 720

Ashman, K. \& Zepf, S., 1992, 384,50

Ashman, K. \& Zepf, S., 2001, ApJ, 122, 1888

Baek, C., Kang, H., Kim, J., Ryu, D., 2005, astro-ph

Barth, A. J., Ho, L. C., Filippenko, A. V., \& Sargent, W. L. W. 1995, AJ, 110, 1009

Bensch, F., Stutzki, J., \& Ossenkopf, V. 2001, A\&A, 366, 636

Blair, W., Kirshner, R., ApJ, 289, 582

Braine, J., \& Combes, F. 1992, A\&A, 264, 433

Brouillet, N., \& Schilke, P. 1993, A\&A, 277, 381

Chernoff, D. \& Weinberg, M., 1990, ApJ, 351, 121

Clark, J., Negeuruela, I., Crowther, P., Goodwin, P., 2005, AA, 434, 949

Davies, R. I., Tacconi, L. J., \& Genzel, R. 2004, ApJ, 602, 148

de Grijs, R., O’Connell, R. W., \& Gallagher, J. S., III. 2001, AJ, 121, 768

Downes, D., \& Solomon, P. M. 1998, ApJ, 507, 615

Elmegreen, B. G. 1983, MNRAS, 203, 1011

Elmegreen, B. G. \& Efremov, Y. 1997, ApJ, 480, 235

Elmegreen, B. G., \& Scalo, J. 2004, ARA\&A, 42, 211

Fall, S. M., \& Rees, M. J. 1985, ApJ, 298, 18

Field, G. B. 1965, ApJ, 142, 531 
Freedman, W. L., et al. 1994, ApJ, 427, 628

Fritze-v. Alvensleben, U. 1999, A\&A, 342, L25

Griffiths, R. E., Ptak, A., Feigelson, E. D., Garmire, G., Townsley, W., Brandt, W. N., Sambruna, R., \& Bregman, J. N. 2000, Science, 290, 17

Harris, W. E., \& Pudritz, R. E. 1994, ApJ, 429, 177

Ho, L. C. 1997, Rev. Mexicana Astron. Astrofis. Conf., 6, 5

Ho, L. C., \& Filippenko, A. V. 1996a, ApJ, 466, L83

—. 1996b, ApJ, 472, 600

Holtzmann, J., et al. 1992, AJ, 103, 691

Israel, F. P. 1997, A\&A, 328, 471

Inutsuka, S. \& Koyama, H., 2004, Rev. Mex. AA, 22, 26

Kennicutt, R. C. 1998, ARA\&A, 36, 189

Kumai, Y., Hashi, Y., \& Fujimoto, M. 1993, ApJ, 416, 576

Lada, C. J., Margulis, M., \& Dearborn, D. 1984, ApJ, 285, 141

Larsen, S. S., \& Richtler, T. 2004, A\&A, 427, 495

Larson, R. B. 1981, MNRAS, 194, 809

Lipscy, S. J., \& Plavchan, P. 2004, ApJ, 603, 82

Mac Low, M.-M., \& Klessen, R. S. 2004, Rev. Mod. Phys., 76, 125

Maoz, D., Barth, A. J., Ho, L. C., Sternberg, A., \& Filippenko, A. V. 2001, AJ, 121, 3048

Maoz, D., Barth, A. J., Sternberg, A., Filippenko, A. V., Ho, L. C., Macchetto, F. D., Rix, H.-W., \& Schneider, D. P. 1996, AJ, 111, 2248

Matsushita, S., Kawabe, R., Kohno, K., Matsumoto, H., Tsuru, T. G., \& Vila-Vilaró, B. 2005, ApJ, 618, 712

Mauersberger, R., Henkel, C., Weiß, A., Peck, A., \& Hagiwara, Y. 2004, A\&A, 403, 561

McKee, C. F., \& Cowie, L. L, 1977, ApJ, 213, 215 
Melo, V. P., Muñoz-Tuñon, C., Maíz-Apellániz, J., \& Tenorio-Tagle, G. 2005, ApJ, 619, 270

Mengel, S., Lehnert, M. D., Thatte, N., \& Genzel, R. 2002, A\&A, 383, 137

Mezger, P. G., \& Henderson, A. P. 1967, ApJ, 147, 471

Meurer, G. R., et al. 1995, AJ, 110, 2665

Murray, S., \& Lin, D., 1989, ApJ 339, 933

Murray, S., \& Lin, D., 1991, ApJ 367, 149

O’Connell, R. W., Gallagher, J. S., Hunter, D. A., \& Colley, W. N. 1995, ApJ, 446, L1

O’Connell, R. W., \& Mangano, J. J. 1978, ApJ, 221, 62

Oike, T., Kawaguchi, K., Takano, S., \& Nakai, N. 2004, PASJ, 56, 431

Peebles, P. J. E., \& Dicke, R. H. 1968, ApJ, 154, 891

Petitpas, G. R., \& Wilson, C. D. 2000, ApJ, 538, L117

Rodríguez-Rico, C. A., Viallefond, F., Zhao, J. H., Goss, W. M., \& Anantharamaiah, K. R. 2004, ApJ, 616, 783

Rosolowsky, E., Engargiola, G., Plambeck, R., \& Blitz, L. 2003, ApJ, 599, 258

Sanders, D. B., \& Mirabel, I. F. 1996 ARA\&A, 34, 749

Satyapal, S., Watson, D. M., Pipher, J. L., Forrest, W. J., Greenhouse, M. A., Smith, H. A., Fischer, J., \& Woodward, C. E. 1997, ApJ, 483, 148

Schmidt, M. 1959, ApJ, 129, 243

Schweizer, F., Miller, B. W., Whitmore, B. C., Fall, S. M. 1996, AJ, 112, 1839

Scoville, N. Z., \& Young, J. S. 1983, ApJ, 265, 148

Soifer, B. T., Sanders, D. B., Madore, B. F., Neugebauer, G., Danielson, G. E., Elias, J. H., Lonsdale, C. J., \& Rice, W. L. 1987, ApJ, 320, 238

Shen, J., \& Lo, K.-Y. 1995, ApJ, 445, L99

Spitzer, L., Jr. 1978, Physical Processes in the Interstellar Medium (New York: Wiley)

Strong, A. W., et al. 1988, A\&A, 207, 1 
Stutzki, J., \& Güsten, R. 1990, ApJ, 356, 513

Vesperini et al. 2003, ApJ 593, 760

Wada, K., \& Tomisaka, K. 2005, ApJ, 619, 93

Wang, M., Henkel, C., Chin, Y.-N., Whiteoak, J. B., Cunningham, M. H., Mauersberger, R., \& Muders, D. 2004, A\&A, 422, 883

Whitmore, B. C., Zhang, Q., Leitherer, C., Fall, S. M., Schweizer, F., \& Miller, B. W. 1999, AJ, 118, 1551

Williams, J. P., de Geus, E. J., \& Blitz, L. 1994, ApJ, 428, 693

Wilson, C. D. 1995, ApJ, 448, L97

Wilson, C. D., Scoville, N., Madden, S. C., \& Charmandaris, V. 2003, ApJ, 599, 1049

Yun, M. S., Ho, P. T. P., \& Lo, K.-Y. 1993, ApJ, 411, L17

Zhang, Q., \& Fall, S. M. 1999, ApJ, 527, L81 

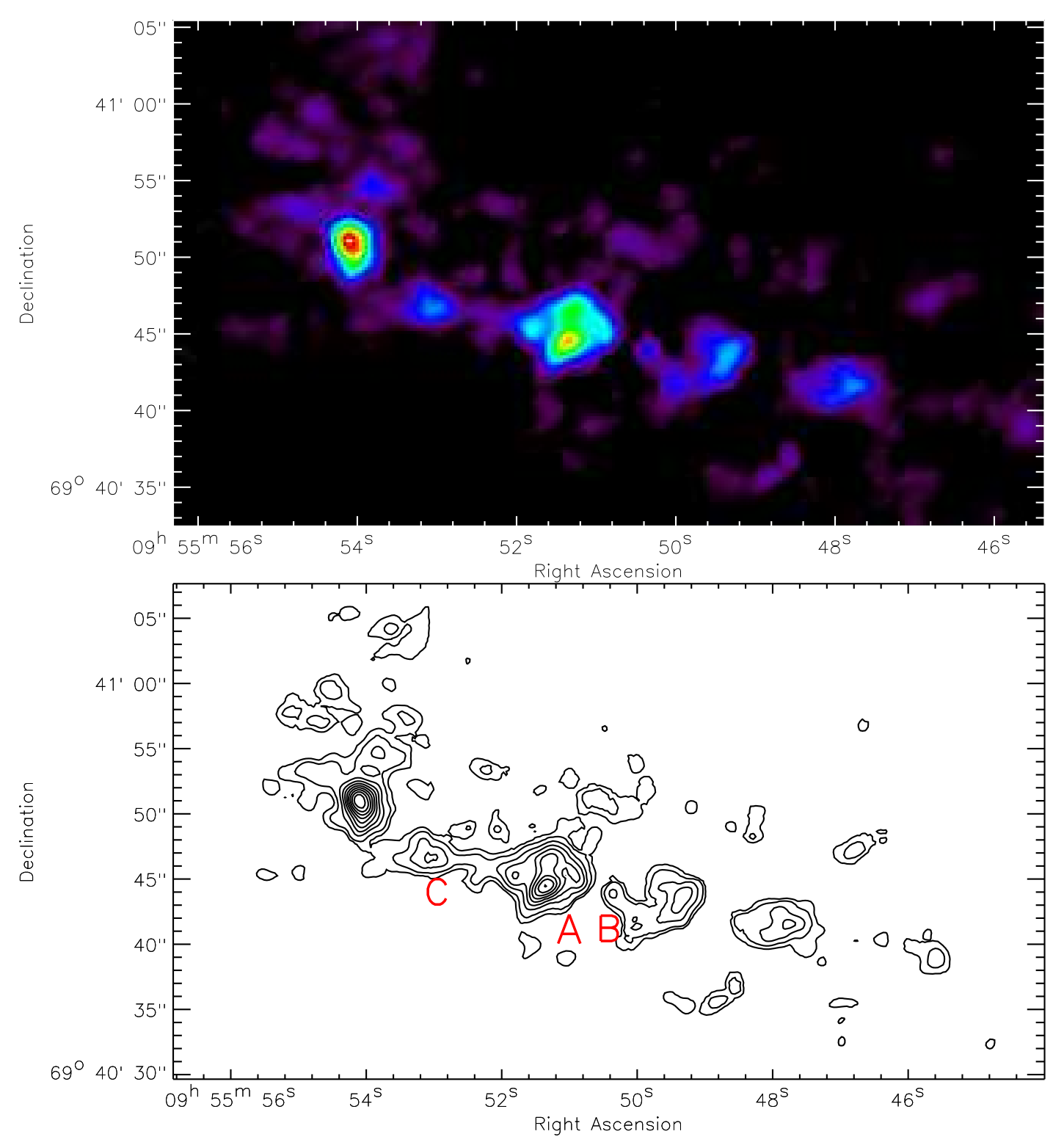

Fig. 1. - The integrated intensity of $\mathrm{CO}(2-1)$ emission in the central region of M82 in color and in contour. The contours are in multiples of $10 \%$ of the peak flux of $8.57 \times 10^{4} \mathrm{Jy} \mathrm{beam}^{-1}$ $\mathrm{km} \mathrm{s}^{-1}$; an extra contour is drawn at the $5 \%$ level. The epoch of the coordinates is J2000. 


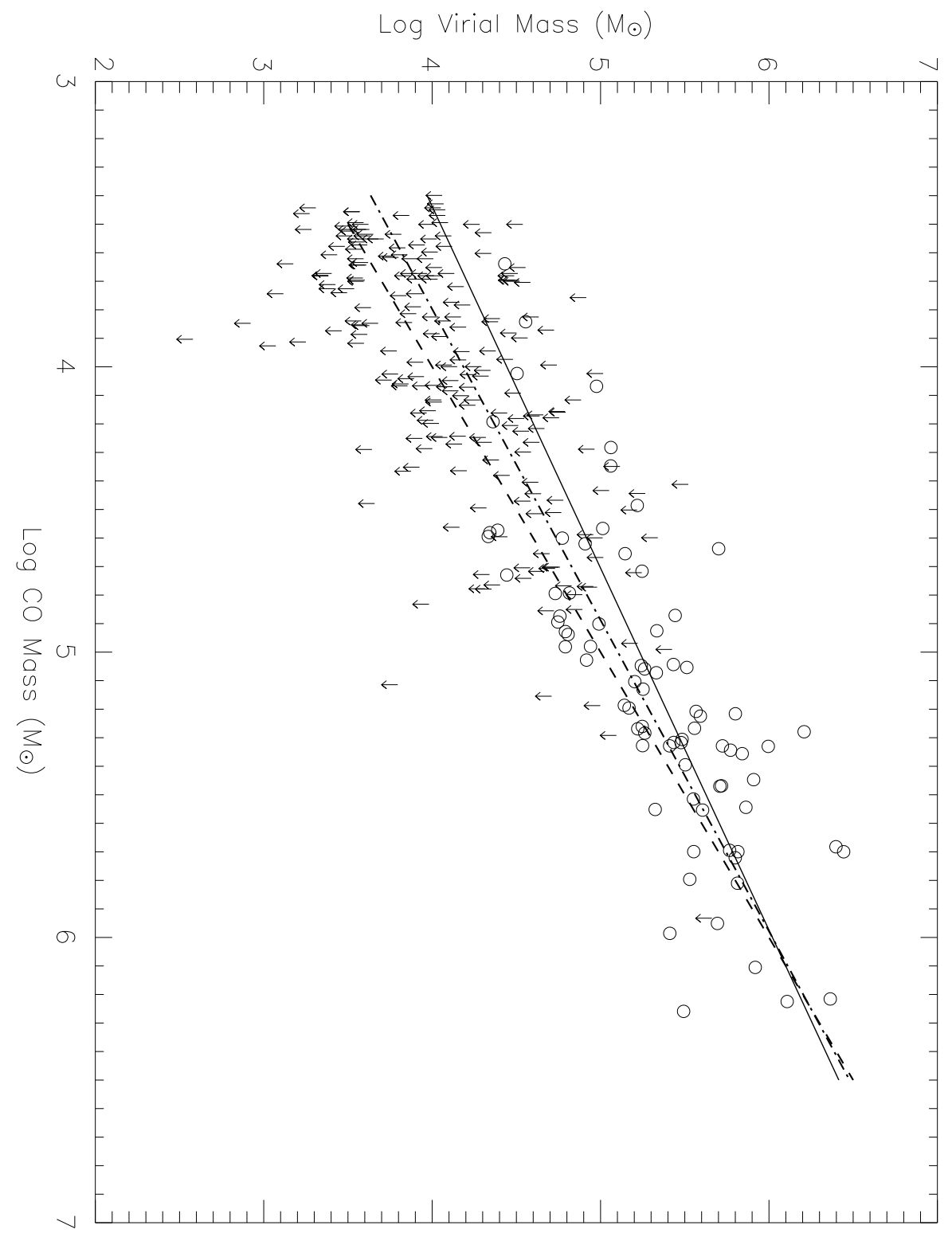

Fig. 2. - The virial mass $\left(M_{\mathrm{vir}}=5 \Delta R \sigma_{v}^{2} / \alpha G\right)$ compared with the mass determined from the CO line brightness. Clouds with unresolved sizes are plotted as upper limits. If the error on the mass of each cloud were $100 \%$, the error bars would have a width of 0.3 in each direction. The dashed line is the line of virial equilibrium (virial mass $=\mathrm{CO}$ mass). The best fit through the masses of the resolved clouds, those with radii larger than the angular resolution, is shown as a solid line while the best fit through all the clouds is a dot-dashed line. 


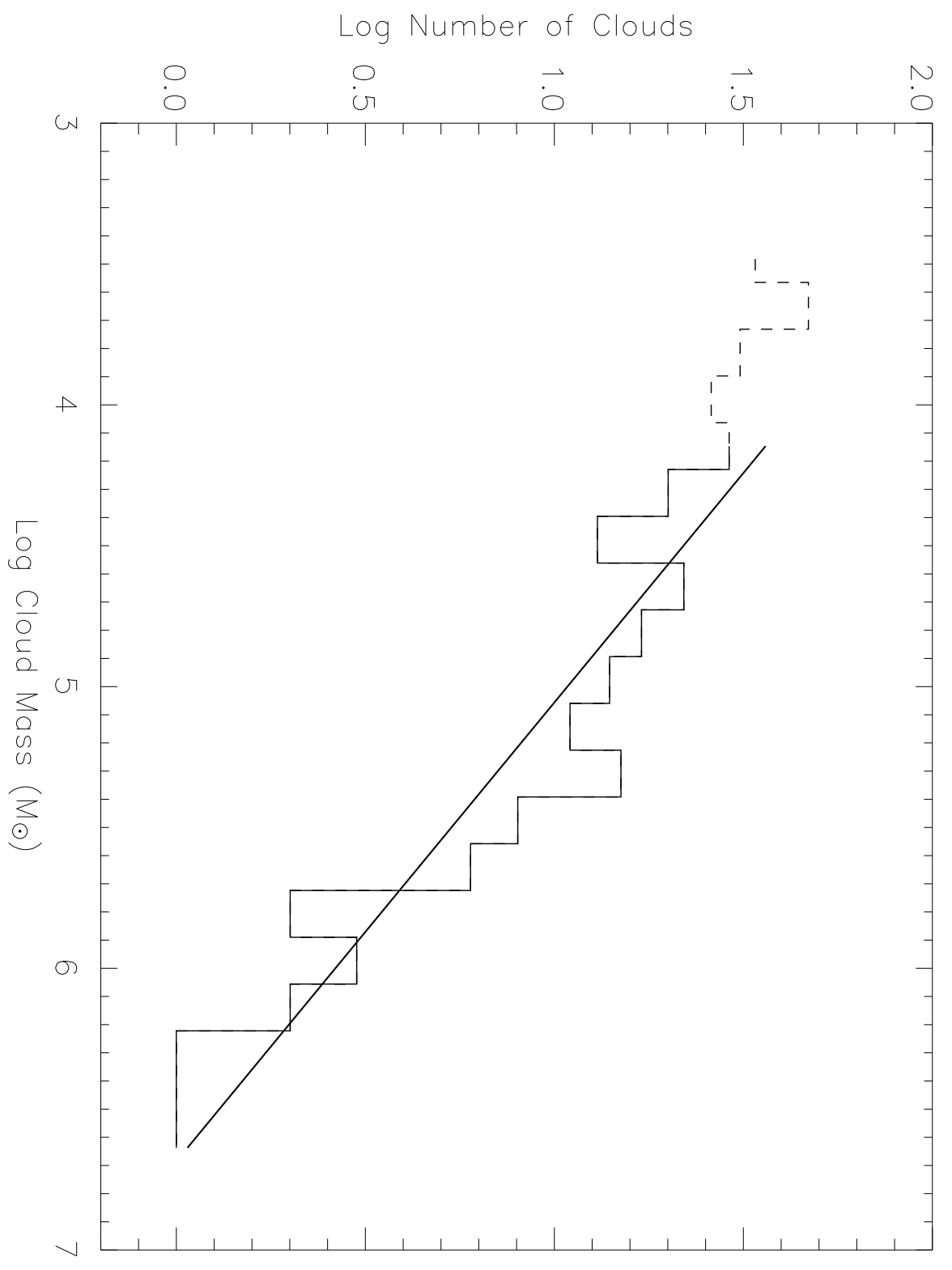

Fig. 3. - The mass spectrum of molecular clouds in the nucleus of M82. The cloud masses were estimated from the CO brightness, and are binned at $\Delta \log M=0.165$ to produce 20 bins across the mass range from 3.4 to $6.7 \log \mathrm{M}_{\odot}$. The heavy solid line is a fit to the M82 data, The data from the dashed portion of the histogram, cloud masses $<10^{4} \mathrm{M}_{\odot}$, was not included in the fit. $d N / d \log M \propto-0.5 \pm 0.04$. 


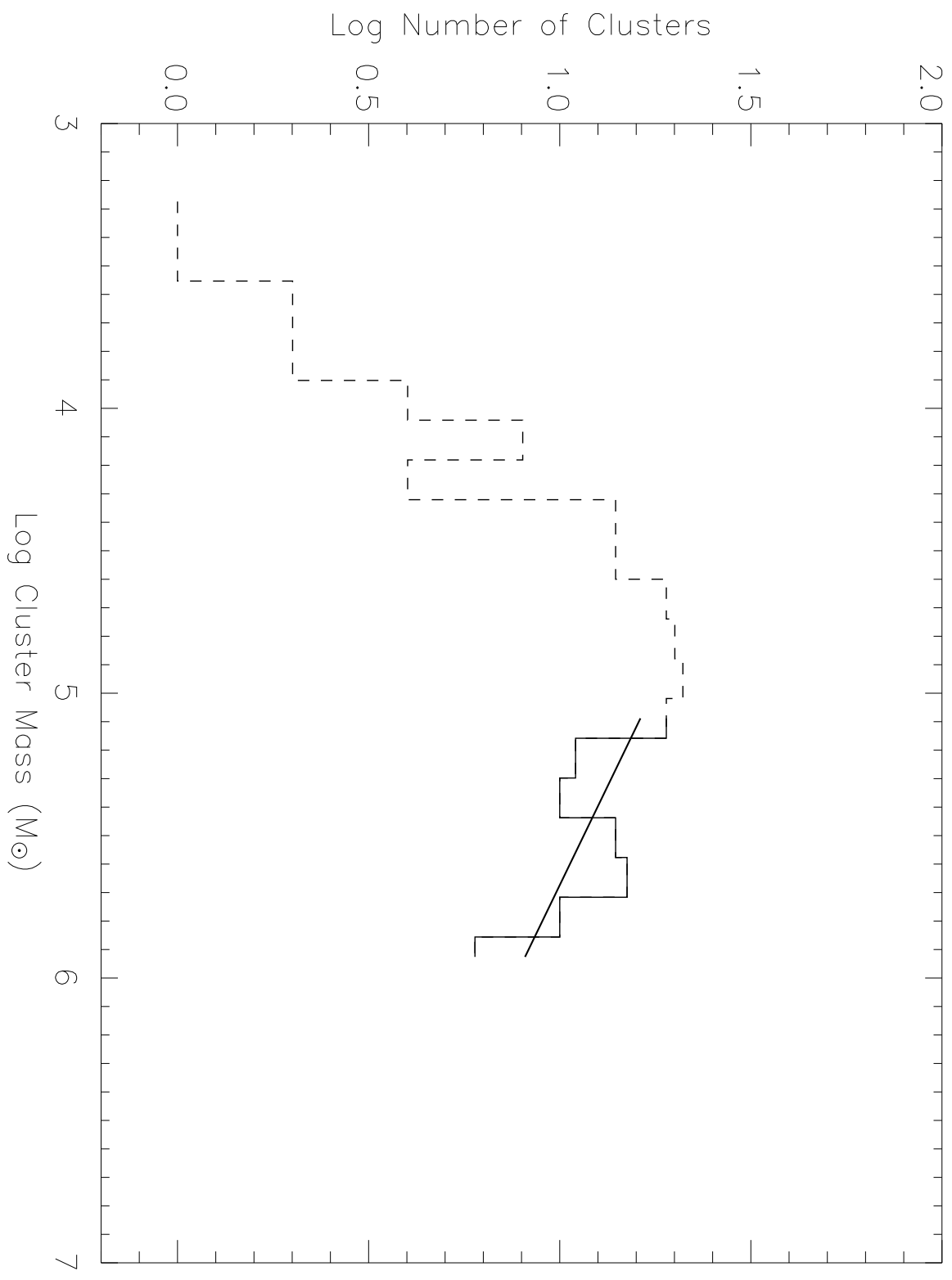

Fig. 4.- The mass spectrum of the SSCs in the nucleus of M82 from Melo et al. (2005). The histogram shows the mass spectrum. The slope of $0.4(\mathrm{dN} / d \log \mathrm{M})$ is fit to the upper end of the mass spectrum where the sample is assumed to be complete. The data on the dashed portion of the histogram were not used in the fitting. 


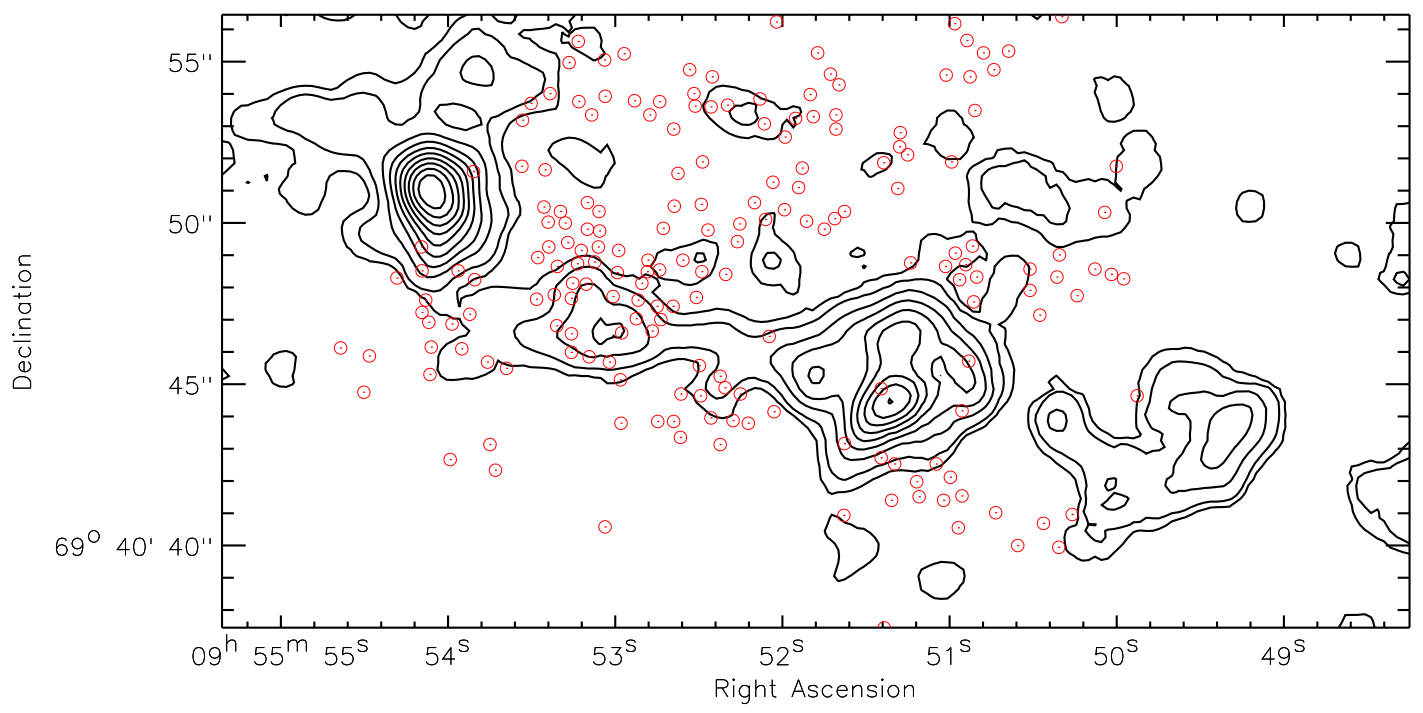

Fig. 5.- The CO(2-1) integrated intensity with young SSCs from Melo et al. (2005). The observations of the SSCs covered only a portion of the center of the galaxy, approximately as outlined by the extent of the SSCs. The epoch of the coordinates is J2000. 


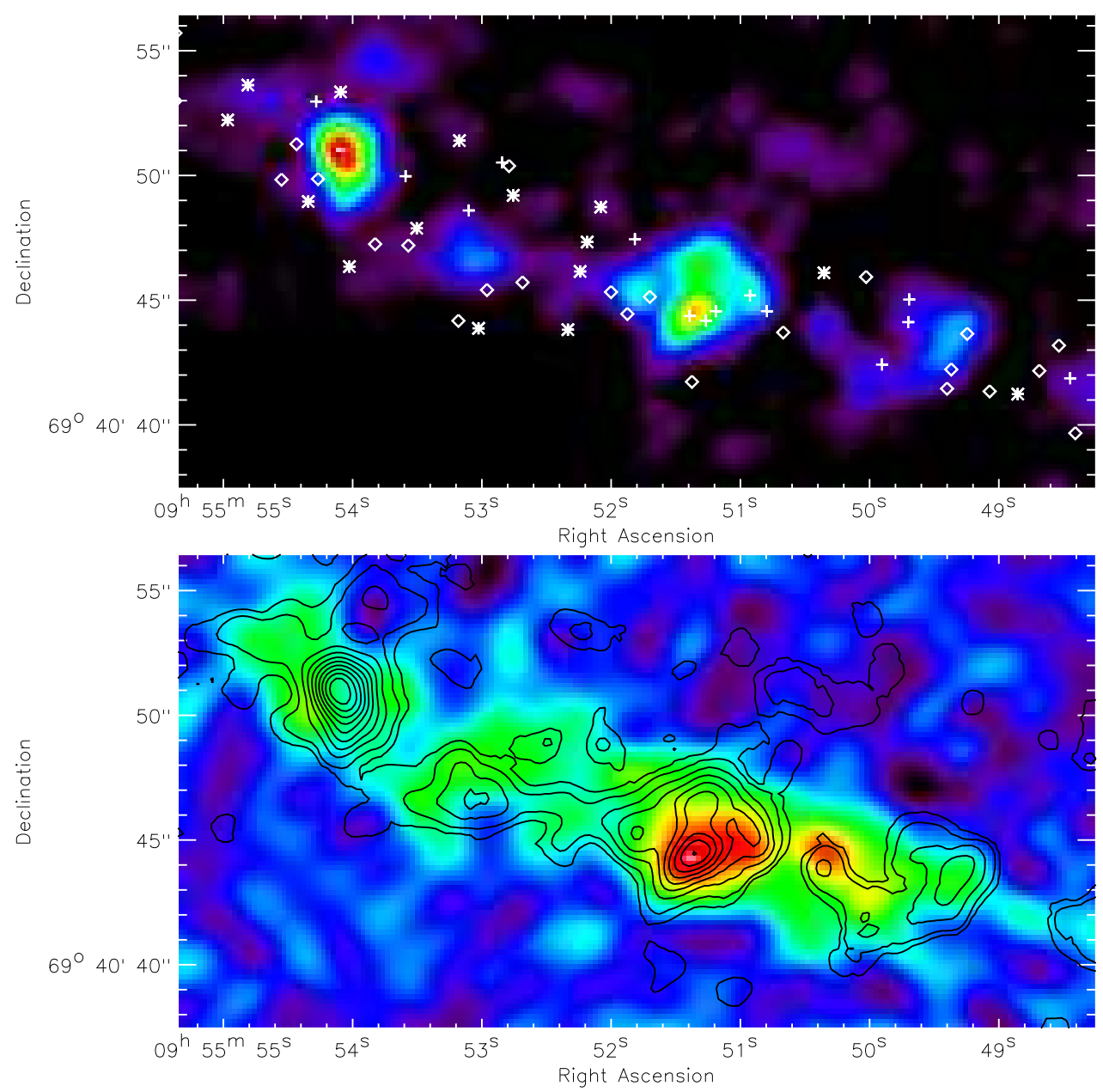

Fig. 6. - The integrated intensity of $\mathrm{CO}(2-1)$ emission in the central region of M82. The symbols show the positions of radio point sources from Rodríguez-Rico et al. (2004). Diamonds represent supernova remnants, crosses represent H II regions, asterisks represent radio point sources of unknown spectral index (either supernova remnants or H II regions).

The integrated intensity of $\mathrm{CO}(2-1)$ in contour and the $100 \mathrm{GHz}$ radio continuum from Matsushita et al. (2005) in color. The $\mathrm{CO}(2-1)$ is generally inversely correlated with the radio point sources not correlated in detail with the extended radio continuum. The epoch of the coordinates is J2000. 


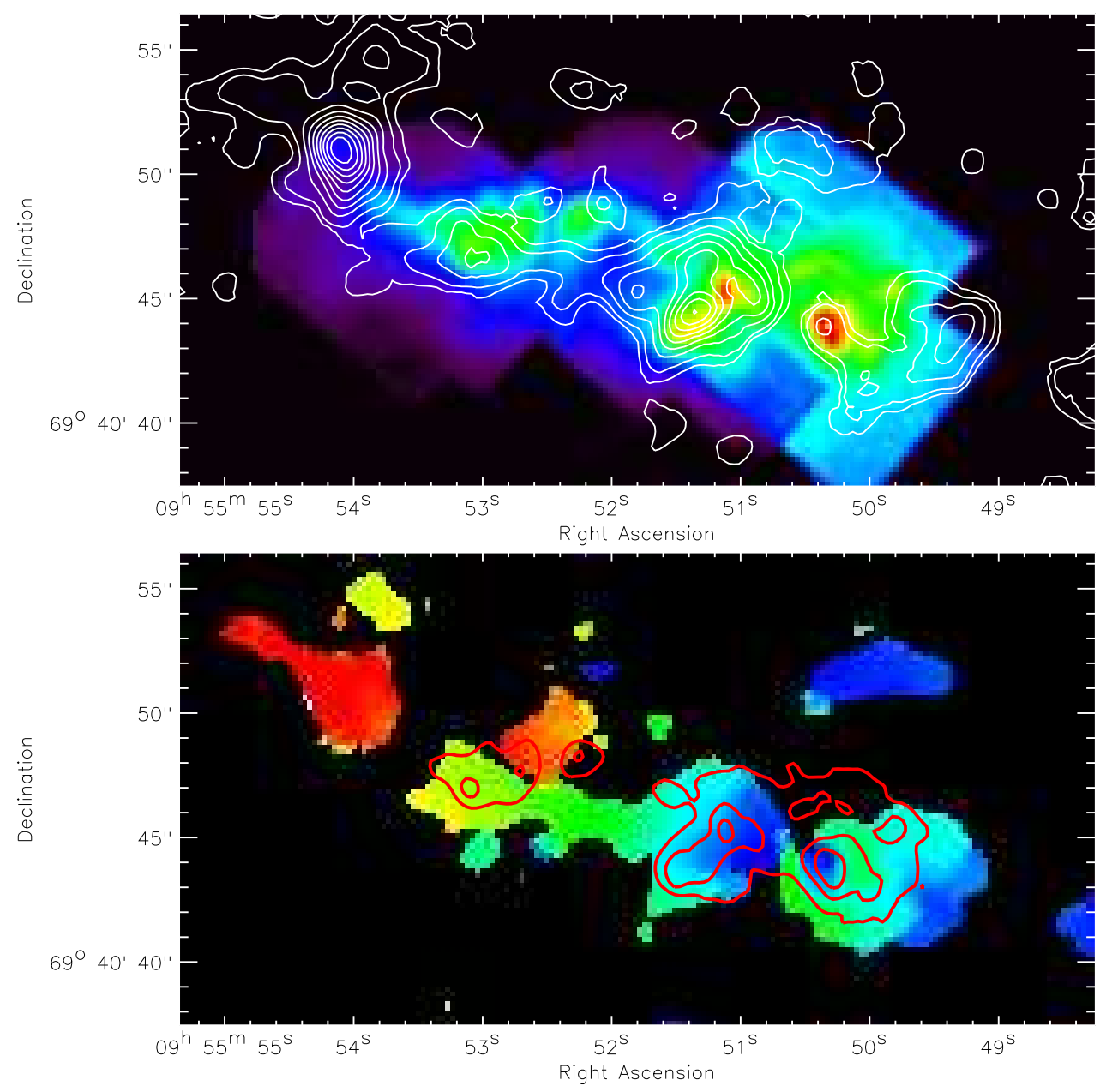

Fig. 7.-

The mid-IR from Lipscy \& Plavchan (2004) in color with contours of integrated CO(2-1) emission. The contour levels are as in figure 1.

Comparison between $\mathrm{CO}(2-1)$ velocity in color and mid-IR emission in contour. The Mid-IR in contour is the same data as the mid-IR in color in the figure above. The epoch of the coordinates is J2000. 


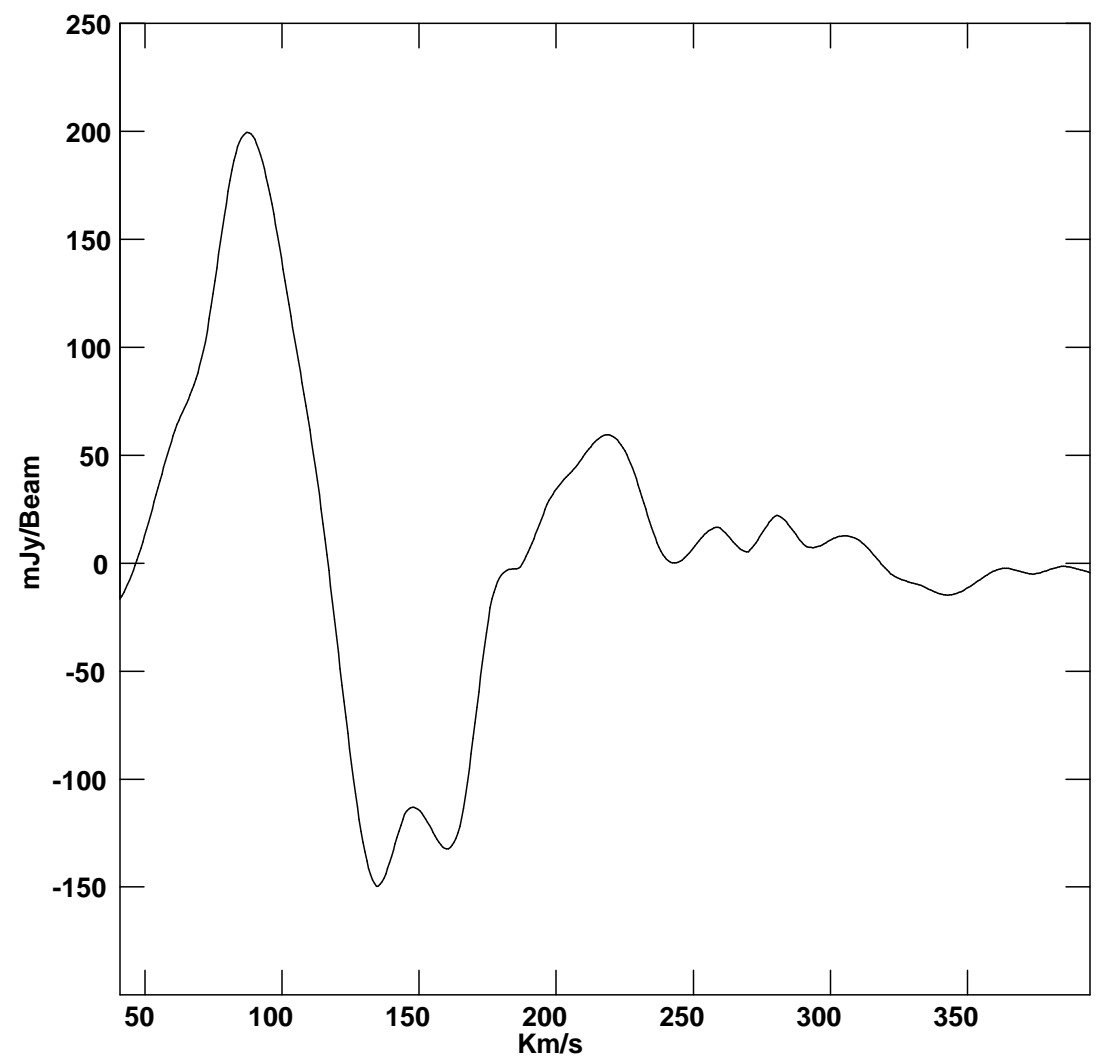

Fig. 8.- CO spectrum at the mid-IR peak in the small cloud. The inverse P-Cygni spectral line profile (absorption red-shifted with respect to the emission) indicates that the gas is moving inward at a speed of about $35 \mathrm{kms}^{-1}$. 


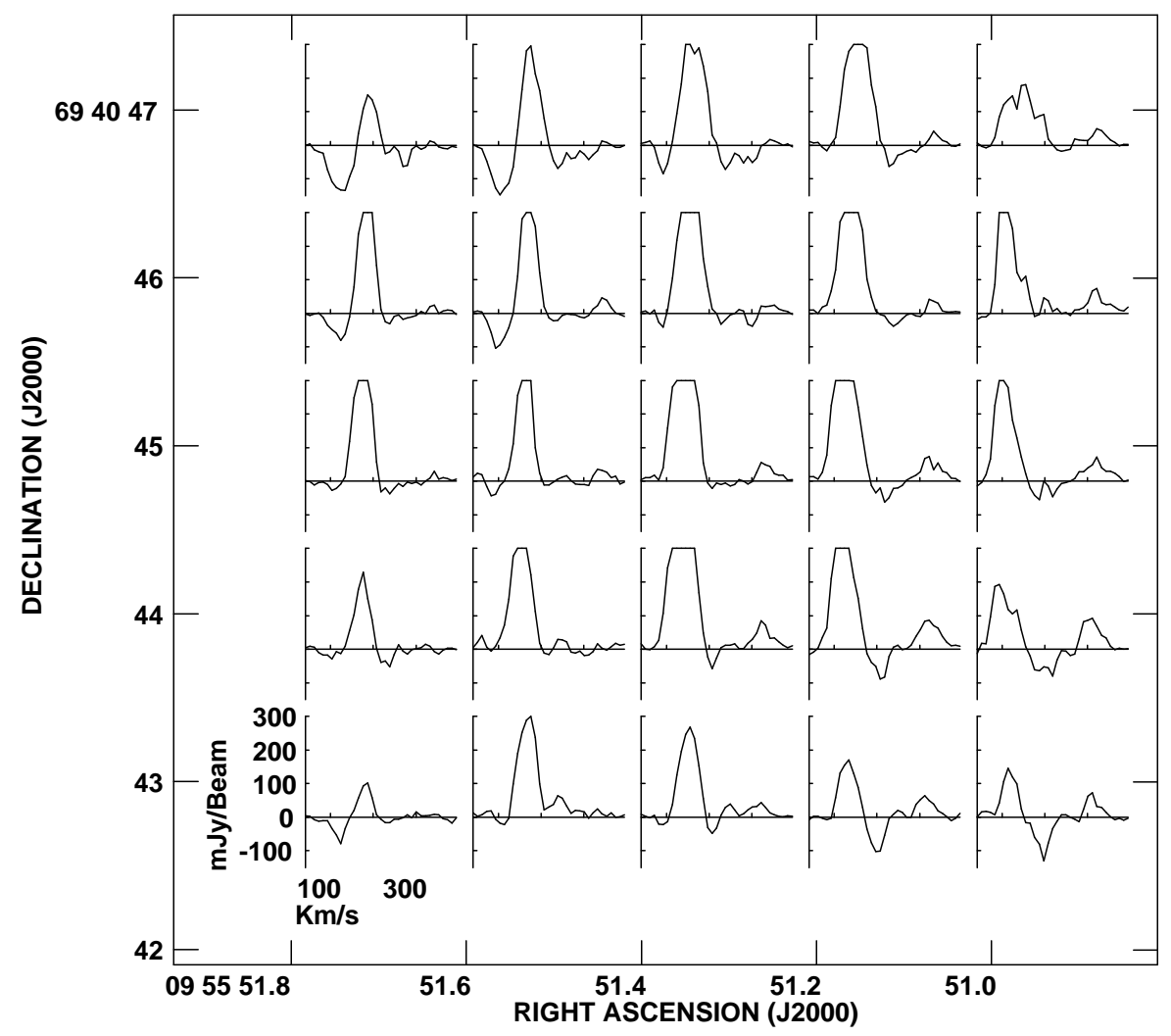

Fig. 9.- CO spectra across the large cloud. The inverse P-Cygni profiles (absorption red-shifted with respect to the emission) seen on the west (right) side of the cloud indicate compression in this region. The classic P-Cygni profiles (absorption blue-shifted with respect to the emission) seen on the east (left) side of the cloud indicate expansion in this east half of the cloud. 


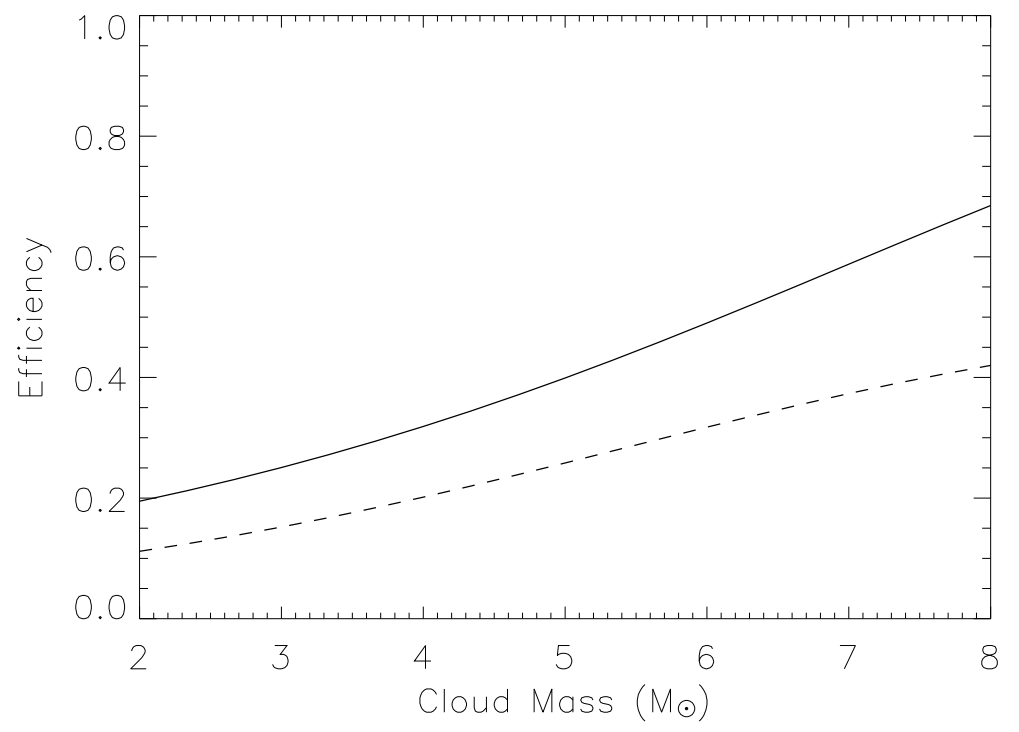

Fig. 10.- The solid line is the calculation exactly from Elmegreen and Efremov (1997) that shows that the efficiency of star formation as a function of the mass of clouds in an environment with a pressure 160 times higher than that in the solar neighborhood. The dashed line is an improved calculation that shows that starting from normal pressure and increasing the pressure over a crossing time lowers the efficiency. High efficiency requires forming the cloud at high pressure or a threshold to prevent star formation at lower pressures. 